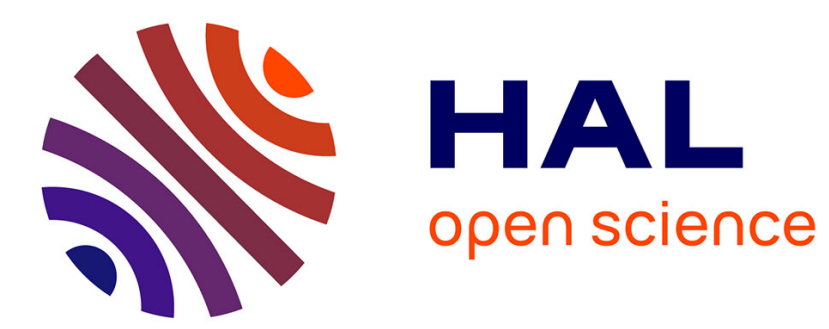

\title{
Penalization method for viscous incompressible flow around a porous thin layer \\ Gilles Carbou
}

\section{To cite this version:}

Gilles Carbou. Penalization method for viscous incompressible flow around a porous thin layer. Nonlinear Analysis: Real World Applications, 2004. hal-01728852

\section{HAL Id: hal-01728852 \\ https://hal.science/hal-01728852}

Submitted on 12 Mar 2018

HAL is a multi-disciplinary open access archive for the deposit and dissemination of scientific research documents, whether they are published or not. The documents may come from teaching and research institutions in France or abroad, or from public or private research centers.
L'archive ouverte pluridisciplinaire HAL, est destinée au dépôt et à la diffusion de documents scientifiques de niveau recherche, publiés ou non, émanant des établissements d'enseignement et de recherche français ou étrangers, des laboratoires publics ou privés. 


\title{
Penalization method for viscous incompressible flow around a
} porous thin layer

\author{
Gilles CARBOU \\ $M A B$, Université Bordeaux 1 \\ 351, Cours de la Libération, 33405 Talence Cedex, France \\ carbou@math.u-bordeaux.fr
}

\section{Introduction}

\subsection{Numerical context}

Penalization methods are now quite classical to compute the flow of an incompressible fluid around a no-slip boundary. The advantage of these methods is to avoid body-fitted unstructured mesh. In this paper, we study a penalization method conceived by C.H. Bruneau and I. Mortazavi. They use this method in [5] to compute the flow around an obstacle surounded by a thin layer of porous material, with applications in passive control for ground vehicles.

Let $\Omega$ be a regular bounded domain of $\mathbb{R}^{3}$ and $\mathcal{O}$ be a regular open subset of $\Omega$ such that $\overline{\mathcal{O}} \subset \Omega$. We denote $\mathcal{U}=\Omega \backslash \overline{\mathcal{O}}$ and $\Gamma=\partial \mathcal{O}$. We fix $\kappa>0$.

For $\varepsilon>0$, we set

$$
\omega_{\varepsilon}=\{x \in \mathcal{O}, \quad 0<\operatorname{dist}(x, \Gamma)<\kappa \varepsilon\}
$$

We denote $\mathcal{U}^{\varepsilon}=\overline{\mathcal{U}} \cup \omega_{\varepsilon}$. The obstacle is represented by $\mathcal{O} \backslash \omega_{\varepsilon}$ and $\omega_{\varepsilon}$ is the thin layer of porous material. We set $\Gamma_{\varepsilon}=\{x \in \mathcal{O}, \operatorname{dist}(x, \Gamma)=\varepsilon\}$.

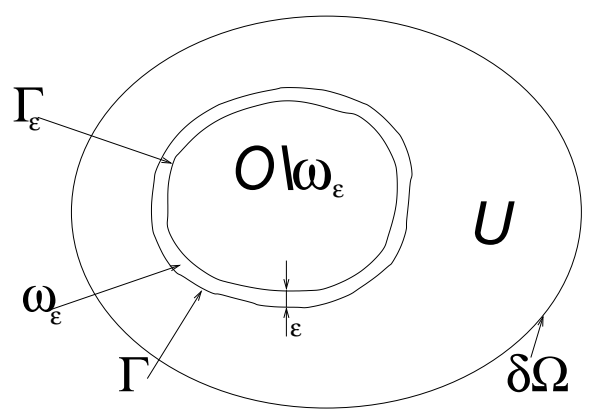

We are interested in the following penalized problem :

$$
\left\{\begin{array}{l}
\frac{\partial u^{\varepsilon}}{\partial t}-\Delta u^{\varepsilon}+\left(u^{\varepsilon} \cdot \nabla\right) u^{\varepsilon}+\frac{1}{\varepsilon} \chi_{\omega_{\varepsilon}} u^{\varepsilon}+\nabla p^{\varepsilon}=f \text { in } \mathcal{U}^{\varepsilon} \\
u^{\varepsilon}=0 \text { on } \partial \mathcal{U}_{\varepsilon} \\
\operatorname{div} u^{\varepsilon}=0 \text { in } \mathcal{U}^{\varepsilon}
\end{array}\right.
$$

where $\chi_{\omega_{\varepsilon}}(x)=1$ if $x \in \omega_{\varepsilon}$ and equals zero if $x \notin \omega_{\varepsilon}$, that is we add to Navier Stokes equation a penalization term of order $\varepsilon^{-1}$ in the thin layer $\omega_{\varepsilon}$ of thikness $\kappa \varepsilon$.

In this paper we first give an asymptotic expansion of $u^{\varepsilon}$ when $\varepsilon$ goes to zero. Furthermore we study another model of porous thin layer wich consists in replacing the equation in the thin 
layer by an equivalent boundary condition on $\Gamma$ :

$$
\left\{\begin{array}{l}
\frac{\partial v^{\varepsilon}}{\partial t}-\Delta v^{\varepsilon}+\left(v^{\varepsilon} \cdot \nabla\right) v^{\varepsilon}+\nabla q^{\varepsilon}=f \text { in } \mathcal{U} \\
v^{\varepsilon}=-\kappa \varepsilon\left(\frac{\partial v^{\varepsilon}}{\partial n}\right)_{T} \text { on } \Gamma
\end{array}\right.
$$

where $\left(\frac{\partial v^{\varepsilon}}{\partial n}\right)_{T}$ is the tangential part of $\frac{\partial v^{\varepsilon}}{\partial n}$ on $\Gamma$.

For this equation we give an asymptotic expansion of $v^{\varepsilon}$ when $\varepsilon$ goes to zero and we prove that $u^{\varepsilon}-v^{\varepsilon}$ is of order $\varepsilon^{2}$.

\section{$1.2 \quad$ Mains Results}

We introduce $\mathbf{V}=\left\{v \in H_{0}^{1}\left(\mathcal{U} ; \mathbb{R}^{3}\right), \quad \operatorname{div} v=0\right\}$.

We recall a proposition partially proved in [7] concerning the Navier Stokes equation around the obstacle $\mathcal{O}$ :

Proposition 1.1 Let $v_{0} \in H^{8}(\mathcal{U}) \cap \mathbf{V}$. Let $f \in \mathcal{C}^{\infty}\left(\mathbb{R}^{+} \times \mathcal{U}\right)$ with space support inclused in $\mathcal{U}$. There exists a time $T^{*}>0$ and there exists $V^{0}$ defined on $\left[0, T^{*}[\times \mathcal{U}\right.$ such that

$$
\left\{\begin{array}{l}
\frac{\partial V^{0}}{\partial t}-\Delta V^{0}+\left(V^{0} \cdot \nabla\right) V^{0}+\nabla p^{0}=f \quad \text { in }\left[0, T^{*}[\times \mathcal{U}\right. \\
\operatorname{div} V^{0}=0 \quad \text { in }\left[0, T^{*}[\times \mathcal{U}\right. \\
V^{0}=0 \quad \text { on }\left[0, T^{*}[\times \partial \mathcal{U}\right. \\
V^{0}(t=0)=v_{0} \quad \text { in } \mathcal{U} .
\end{array}\right.
$$

For all $T<T^{*}$, this solution $V^{0}$ is in $L^{\infty}\left(0, T ; H^{8}(\mathcal{U})\right) \cap L^{2}\left(0, T ; H^{9}(\mathcal{U})\right)$.

Remark 1.1 In the two-dimensional case we can prove that $T^{*}=+\infty$.

We consider the following penalized thin layer problem :

$$
\left\{\begin{array}{lr}
\frac{\partial u^{\varepsilon}}{\partial t}-\Delta u^{\varepsilon}+\left(u^{\varepsilon} \cdot \nabla\right) u^{\varepsilon}+\nabla \pi^{\varepsilon}+\frac{1}{\varepsilon} \chi_{\omega_{\varepsilon}} u^{\varepsilon}=f & \text { in }\left[0, T^{*}\left[\times \mathcal{U}_{\varepsilon}\right.\right. \\
\operatorname{div} u^{\varepsilon}=0 & \text { in }\left[0, T^{*}\left[\times \mathcal{U}_{\varepsilon}\right.\right. \\
u^{\varepsilon}=0 & \text { on }\left[0, T^{*}\left[\times \partial \mathcal{U}_{\varepsilon}\right.\right. \\
u^{\varepsilon}(0, x)=u_{0}^{\varepsilon}(x) & \text { in } \mathcal{U}_{\varepsilon} .
\end{array}\right.
$$

For $x \in \omega$, we introduce $\varphi(x)=\operatorname{dist}(x, \Gamma)$ and $P(x)$ the orthogonal projection of $x$ onto $\Gamma$. We remark that since $\Gamma$ is a regular surface of $\mathbb{R}^{3}, \varphi$ and $P$ are regular in a neigbourhood of $\Gamma$.

For well prepared initial data, we obtain an asymptotic expansion of $u^{\varepsilon}$ decribed in the following theorem : 
Theorem 1.1 Let $v_{0}, f, V^{0}$ and $T^{*}$ as in Proposition 1.1.

There exists two profiles $V^{1}:\left[0, T^{*}\left[\times \mathcal{U} \longrightarrow \mathbb{R}^{3}\right.\right.$ and $W^{1}:\left[0, T^{*}\left[\times \Gamma \times[0,1] \longrightarrow \mathbb{R}^{3}\right.\right.$ such that if $u_{0}^{\varepsilon}$ is an initial data of the form :

$$
u_{0}^{\varepsilon}(x)=\left\{\begin{array}{l}
v_{0}(x)+\varepsilon V^{1}(0, x)+\varepsilon^{2} r^{\varepsilon}(x) \text { if } x \in \mathcal{U} \\
\varepsilon W^{1}\left(0, P(x), \frac{\varphi(x)}{\varepsilon}\right)+\varepsilon^{2} r^{\varepsilon}(x) \text { if } x \in \omega_{\varepsilon}
\end{array}\right.
$$

where $\left\|r^{\varepsilon}\right\|_{L^{2}\left(\mathcal{U}_{\varepsilon}\right)} \leq K$ and such that $\operatorname{div} u_{0}^{\varepsilon}=0$ on $\mathcal{U}_{\varepsilon}$, then there exists $u^{\varepsilon}$ a solution of the penalized problem (1.1) which satisfies

$$
u^{\varepsilon}(t, x)=\left\{\begin{array}{l}
V^{0}(t, x)+\varepsilon V^{1}(t, x)+\varepsilon^{2} v_{\varepsilon}^{r}(t, x) \text { for } x \in \mathcal{U} \\
\varepsilon W^{1}\left(t, P(x), \frac{\varphi(x)}{\varepsilon}\right)+\varepsilon^{2} w_{\varepsilon}^{r}(t, x) \text { for } x \in \omega_{\varepsilon}
\end{array}\right.
$$

where $v_{\varepsilon}^{r}$ and $w_{\varepsilon}^{r}$ are bounded in $L^{\infty}\left(0, T ; L^{2}\right) \cap L^{2}\left(0, T ; H^{1}\right), \forall T<T^{\star}$.

In order to perform the asymptotic expansion of $u^{\varepsilon}$ we will use a BKW method, that is we will formally write $u^{\varepsilon}$ on the form of its ansatz :

$$
u^{\varepsilon}(t, x)=\left\{\begin{array}{l}
V^{0}(t, x)+\varepsilon V^{1}(t, x)+\varepsilon^{2} V^{2}(t, x)+\varepsilon^{3} V^{3}(t, x) \text { if } x \in \mathcal{U} \\
\varepsilon W^{1}\left(t, P(x), \frac{\varphi(x)}{\varepsilon}\right)+\varepsilon^{2} W^{2}\left(t, P(x), \frac{\varphi(x)}{\varepsilon}\right)+\varepsilon^{3} W^{3}\left(t, P(x), \frac{\varphi(x)}{\varepsilon}\right) \text { if } x \in \omega_{\varepsilon}
\end{array}\right.
$$

and we will plot this ansatz in Equations (1.1). Then we will identify the different powers of $\varepsilon$ to characterize each term of the ansatz.

Remark 1.2 We will see that in order to obtain a remainder term of order $\varepsilon^{2}$ we have to perform the formal asymptotic expansion at order $\varepsilon^{3}$. This phenomenon is quite classical in this type of problems (see [6] and [8] for example).

Remark 1.3 Each term $V^{i}$ is deduced from the value on $\Gamma$ of $\frac{\partial V^{i-1}}{\partial n}$. It is the reason why we need a lot of regularity on $V^{0}$ and thus on the initial data $v^{0}$. Here is the weakness of $B K W$ method : it is very expensive in regularity.

Remark 1.4 We will see that the profile $V^{1}$ is charaterized by :

$$
\left\{\begin{array}{l}
\frac{\partial V^{1}}{\partial t}-\Delta V^{1}+V^{0} \cdot \nabla V^{1}+V^{1} \cdot \nabla V^{0}+\nabla p^{1}=0 \quad \text { in } \mathbb{R}^{+} \times \mathcal{U} \\
\operatorname{div} V^{1}=0 \quad \text { in } \mathbb{R}^{+} \times \mathcal{U}, \\
V^{1}(t, x)=-\kappa\left(\frac{\partial V^{0}}{\partial n}\right)_{T} \quad \text { on } \mathbb{R}^{+} \times \partial \omega
\end{array}\right.
$$

where $\left(\frac{\partial V^{0}}{\partial n}\right)_{T}=\frac{\partial V^{0}}{\partial n}-\left(\frac{\partial V^{0}}{\partial n} \cdot n\right) n$ is the tangential part of $\frac{\partial V^{0}}{\partial n}$. 
Furthermore $W^{1}$ is given by the expression :

$$
W^{1}(t, \sigma, z)=(z-\kappa)\left(\frac{\partial V^{0}}{\partial n}\right)_{T}(t, \sigma) .
$$

In addition we remark that $W^{1}$ is tangential to $\Gamma$.

Remark 1.5 In the penalized thin layer, the principal terms of the anzatz satisfy an approximation of Brinkmann equation, that is

$$
-\Delta u+\frac{1}{\varepsilon} u+\nabla p \text { is small in } \omega_{\varepsilon}
$$

The phenomenon has been remarked by Khadra and all (see [12]).

We study now a physical model for the flow of an incompressible fluid around a porous obstacle. It consists in computing the flow in the fluid $\mathcal{U}$ and in giving an equivalent boundary condition on $\Gamma$ :

$$
\begin{cases}\frac{\partial v^{\varepsilon}}{\partial t}-\Delta v^{\varepsilon}+\left(v^{\varepsilon} \cdot \nabla\right) v^{\varepsilon}+\nabla p^{\varepsilon}=f & \text { in }\left[0, T^{\star}[\times \mathcal{U}\right. \\ \operatorname{div} v^{\varepsilon}=0 & \text { in }\left[0, T^{\star}[\times \mathcal{U}\right. \\ v^{\varepsilon}=0 & \text { in }\left[0, T^{\star}[\times \partial \Omega\right. \\ v^{\varepsilon} \cdot n=0 & \text { in }\left[0, T^{\star}[\times \Gamma\right. \\ v^{\varepsilon}=-\kappa \varepsilon\left(\frac{\partial v^{\varepsilon}}{\partial n}\right)_{T} & \text { in }\left[0, T^{\star}[\times \Gamma\right. \\ v^{\varepsilon}(0, x)=v_{0}^{\varepsilon}(x) & \text { in } \mathcal{U}\end{cases}
$$

Remark 1.6 This model is obtained by Mikelic in [14], using an homogeneization process in the porous material.

We will prove that this model is equivalent to the thin layer penalization problem since we have the following theorem :

Theorem 1.2 Let $u_{0}, V^{0}$ and $T^{*}$ as in Proposition 1.1. Let $V^{1}$ given by Theorem 1.1. Then if $v_{0}^{\varepsilon}$ is an initial data of the form :

$$
v_{0}^{\varepsilon}(x)=u_{0}(x)+\varepsilon V^{1}(0, x)+\varepsilon^{2} r^{\varepsilon}(x) \text { if } x \in \mathcal{U}
$$

with $\left\|r^{\varepsilon}\right\|_{L^{2}(\mathcal{U})} \leq K$ and such that $\operatorname{div} u_{0}^{\varepsilon}=0$ on $\mathcal{U}$, then there exists $v^{\varepsilon}$ a solution of the problem (1.2) which satisfies

$$
v^{\varepsilon}(t, x)=V^{0}(t, x)+\varepsilon V^{1}(t, x)+\varepsilon^{2} v_{\varepsilon}^{r}(t, x),
$$

where $v_{\varepsilon}^{r}$ is bounded in $L^{\infty}\left(0, T ; L^{2}(\mathcal{U})\right) \cap L^{2}\left(0, T ; H^{1}(\mathcal{U})\right), \forall T<T^{\star}$.

Since $u^{\varepsilon}$ and $v^{\varepsilon}$ have the same asymptotic expansion, we have the following result :

Corollary 1.1 The error between the penalized thin layer solution and the solution of Equation (1.2) is of order $\varepsilon^{2}$ in $L^{\infty}\left(0, T ; L^{2}(\mathcal{U})\right) \cap L^{2}\left(0, T ; H^{1}(\mathcal{U})\right)$. 
This paper is organized as follows.

In the second part we explain the geometrical tools used for the study of the thin layer $\omega_{\varepsilon}$. Indeed we seek the profiles in the coordinates $(P(x), \varphi(x)) \in \Gamma \times[0,1]$ and we have to express the differential operators in these coordinates.

All this work will allow us to prove an important lemma of relevement, that is : if $g \in L^{2}\left(\omega_{\varepsilon}\right)$ with $\int_{\omega_{\varepsilon}} g=0$, there exists $\Psi_{\varepsilon} \in H_{0}^{1}\left(\omega_{\varepsilon}\right)$ such that $\operatorname{div} \Psi_{\varepsilon}=g$ and we can estimate $\Psi_{\varepsilon}$ since there exists a constant $C$ independant on $\varepsilon$ such that $\left\|\psi_{\varepsilon}\right\|_{H_{0}^{1}\left(\omega_{\varepsilon}\right)} \leq \frac{C}{\varepsilon}\|g\|_{L^{2}\left(\omega_{e}\right)}$.

In the third part, using BKW method we characterize the different profiles of the asymptotic expansion of $u^{\varepsilon}$. Next we prove existence and regularity of these profiles, and it is here that we will see why we need a so big regularity for the initial data $v_{0}$.

In the fourth part we estimate the remainder term of the asymptotic expansion using a classical Gronwall Lemma and we conclude the proof of Theorem 1.1.

The fifth part is devoted to the proof of Theorem 1.2. BKW method for this problem and the proof of the regularity for the profiles are already done in the previous section and we only have to estimate the remainder term for this equation.

At last, in the Appendix, we detail the calculations of the second part, for the interested reader.

Remark 1.7 In a previous paper, Carbou and Fabrie study a penalization method without effect of thin layer, i.e. the penalization occurs on the whole domain $\mathcal{O}$, but is numericaly smaller (of order $\left.10^{-8}\right)$. They prove in this case that the error between the penalized problem and the physical obstacle problem is of order $\sqrt{\varepsilon}$. The proof of this result is based on an asymptotic expansion of the solution which describes the boundary layer in the penalized obstacle.

\section{Tools for the study of thin layers}

We will use the following notations :

- $(p \cdot q)$ is the scalar product in $\mathbb{R}^{3}$.

- $\Gamma=\partial \mathcal{O}$,

- for $\sigma \in \Gamma, n(\sigma)$ is the unitary normal to $\Gamma$ at the point $\sigma$, entering in $\mathcal{O}$,

- for $\sigma \in \Gamma, T_{\sigma} \Gamma$ is the tangent plane of $\Gamma$ at the point $\sigma$ :

$$
T_{\sigma} \Gamma=(n(\sigma))^{\perp}
$$

- $\varphi(x)=\operatorname{dist}(x, \Gamma)$ for $x \in \mathcal{O}$,

- $P(x)$ the orthogonal projection of $x$ onto $\Gamma$, for $x \in \omega \mathcal{O}$,

- $\omega_{\varepsilon}=\{x \in \mathcal{O}, \quad 0<\varphi(x)<\kappa \varepsilon\}$,

- for $s>0, \Gamma_{s}=\{x \in \mathcal{O}, \quad \varphi(x)=\kappa s\}$.

\subsection{Geometrical tools}

For $\eta_{0}>0$ small enough, we define a parametrization of $\omega_{\eta_{0}}$ by :

$$
\begin{aligned}
\Psi: \Gamma \times] 0, \kappa \eta_{0}[ & \longrightarrow \omega_{\eta_{0}} \\
\sigma, z & \mapsto \sigma+z n(\sigma)
\end{aligned}
$$


Since $\Gamma=\partial \omega$ is a regular compact surface of $\mathbb{R}^{3}$ without boundary, there exists $\eta_{0}>0$ such that $\Psi$ is a $\mathcal{C}^{\infty}$-diffeomorphism from $\left.\Gamma \times\right] 0, \kappa \eta_{0}\left[\right.$ onto $\omega_{\eta_{0}}$. We remark that for $\varepsilon<\eta_{0}$ the restriction of $\Psi$ to $\Gamma \times] 0, \kappa \varepsilon\left[\right.$ is a $\mathcal{C}^{\infty}$-parametrization of $\omega_{\varepsilon}$.

Furthermore $\varphi$ and $P$ are regular on $\omega_{\eta_{0}}$ and

$$
\forall x \in \omega_{\eta_{0}}, \quad \nabla \varphi(x)=n(P(x)) .
$$

We are lead to precise the expression of the differential operators in the coordinates $(\sigma, z)$.

On the submanifold $\Gamma$ we can classicaly define the integrale and the differential operators $\nabla_{\Gamma}$, $\operatorname{div}_{\Gamma}$ and $\Delta_{\Gamma}$ (see the Appendix for the expression of these operators in a coordinate map). Furthermore, $n$ is a map defined from $\Gamma$ with values in the unit sphere $S^{2}$ so for $\sigma \in \Gamma$, the differential $d n(\sigma)$ is a linear map from $T_{\sigma} \Gamma$ into $T_{n(\sigma)} S^{2}$ and since $T_{n(\sigma)} S^{2}=T_{\sigma} \Gamma$, we can consider $d n(\sigma)$ as an endomorphism of $T_{\sigma} \Gamma$.

Integration : we set for $s \in\left[0, \kappa \eta_{0}[\right.$ and $\sigma \in \Gamma$ :

$$
\gamma_{s}(\sigma)=\operatorname{det}(I d+s d n(\sigma)) .
$$

If $u: \omega_{\eta_{0}} \longrightarrow \mathbb{R}$, denoting $\tilde{u}=u \circ \Psi$, we have :

$$
\int_{\omega_{\eta_{0}}} u=\int_{0}^{\kappa \eta_{0}} \int_{\Gamma} \tilde{u}(\sigma, s) \gamma_{s}(\sigma) d \sigma d s
$$

Gradient : for $\tilde{v}: \Gamma \longrightarrow \mathbb{R}$, we define :

$$
\nabla_{\Gamma_{s}} \tilde{v}(\sigma)=(I d+s d n(\sigma))^{-1}\left(\nabla_{\Gamma} \tilde{v}(\sigma)\right)
$$

and if $u: \omega_{\eta_{0}} \longrightarrow \mathbb{R}$, denoting $\tilde{u}=u \circ \Psi$, we have :

$$
\nabla u(x)=\frac{\partial \tilde{u}}{\partial z}(P(x), \varphi(x)) n(P(x))+\left(\nabla_{\Gamma_{\varphi(x)}} \tilde{u}\right)(P(x), \varphi(x)) .
$$

Divergence Operator : let $\tilde{Y}: \Gamma \longrightarrow T \Gamma$ be a tangent vector field defined on $\Gamma$. We define :

$$
\operatorname{div}_{\Gamma_{s}} \tilde{Y}(\sigma)=\frac{1}{\gamma_{s}(\sigma)} \operatorname{div}{ }_{\Gamma}\left[\gamma_{s}(I d+s d n)^{-1} \tilde{Y}\right](\sigma)
$$

and if $Z: \omega_{\eta_{0}} \longrightarrow \mathbb{R}^{3}$, denoting $\widetilde{Z}=Z \circ \Psi$, we have

$$
\operatorname{div} Z(x)=\frac{\partial \widetilde{Z}_{N}}{\partial z}(P(x), \varphi(x))+G_{\varphi(x)}(P(x)) \widetilde{Z}_{N}(P(x), \varphi(x))+\left(\operatorname{div}_{\Gamma_{\varphi(x)}} \widetilde{Z}_{T}\right)(P(x), \varphi(x))
$$

where $\widetilde{Z}_{N}(\sigma, z)=(\widetilde{Z}(\sigma, z) \cdot n(\sigma))$ is the normal part of $\tilde{Z}$ and $\widetilde{Z}_{T}(\sigma, z)=\widetilde{Z}(\sigma, z)-\widetilde{Z}_{N}(\sigma, z) n(\sigma)$ is its tangential part, and where :

$$
G_{s}(\sigma)=\frac{1}{\gamma_{s}(\sigma)} \frac{\partial \gamma_{s}}{\partial s}(\sigma)
$$

Laplace operator : for $\tilde{v}: \Gamma \longrightarrow \mathbb{R}$ we define

$$
\Delta_{\Gamma_{s}} \tilde{v}=\operatorname{div} \Gamma_{s} \nabla_{\Gamma_{s}} \tilde{v}
$$

and if $u: \omega_{\eta_{0}} \longrightarrow \mathbb{R}$, denoting $\tilde{u}=u \circ \Psi$, we have :

$$
\Delta u(x)=\frac{\partial^{2} \tilde{u}}{\partial z^{2}}(P(x), \varphi(x))+G_{\varphi(x)}(P(x)) \frac{\partial \tilde{u}}{\partial z}(P(x), \varphi(x))+\left(\Delta_{\Gamma_{\varphi(x)}} \tilde{u}\right)(P(x), \varphi(x)) .
$$

Remark 2.1 All these expressions in the new coordinates are proved in the appendix. 


\subsection{Functionnal spaces in thin layers}

In this subsection we precise the dependance on $\varepsilon$ of the Sobolev constants on $H^{1}\left(\omega_{\varepsilon}\right)$. The dependance on $\varepsilon$ of these Sobolev constant acts a crucial part in the estimates of Section 4.

Proposition 2.1 There exists a constant $C$ such that for all $\varepsilon<\eta_{0}$, for all $u \in H^{1}\left(\omega_{\varepsilon}\right)$,

$$
\begin{aligned}
& \|u\|_{L^{6}\left(\omega_{\varepsilon}\right)} \leq \frac{C}{\varepsilon^{\frac{1}{3}}}\left(\|u\|_{L^{2}\left(\omega_{\varepsilon}\right)}+\|\nabla u\|_{L^{2}\left(\omega_{\varepsilon}\right)}\right) \\
& \|u\|_{L^{3}\left(\omega_{\varepsilon}\right)} \leq \frac{C}{\varepsilon^{\frac{1}{6}}}\left(\|u\|_{L^{2}\left(\omega_{\varepsilon}\right)}+\|\nabla u\|_{L^{2}\left(\omega_{\varepsilon}\right)}\right)^{\frac{1}{2}}\|u\|_{L^{2}\left(\omega_{\varepsilon}\right)}^{\frac{1}{2}} \\
& \|u\|_{L^{2}(\Gamma)} \leq C\|u\|_{L^{2}\left(\omega_{\varepsilon}\right)}^{\frac{1}{2}}\left(\frac{1}{\sqrt{\varepsilon}}\|u\|_{L^{2}\left(\omega_{\varepsilon}\right)}^{\frac{1}{2}}+\|\nabla u\|_{L^{2}\left(\omega_{\varepsilon}\right)}^{\frac{1}{2}}\right)
\end{aligned}
$$

Proof : using an atlas of maps covering $\Gamma$ and a partition of the unity, we have to prove the different inequalities in $U \times] 0, \kappa \varepsilon\left[\right.$ where $U$ is an open bounded set of $\mathbb{R}^{2}$ where the map is defined.

The first inequality is proved in Ladyzenskaya (see [13]) and in Teman Ziane (see [15]). From this result and using a classical interpolation inequality between $L^{2}$ and $L^{6}$ we deduce easily the second inequality.

For the last estimate, we consider $u \in \mathcal{D}\left(\overline{\omega_{\varepsilon}}\right)$, and we set $\tilde{u}=u \circ \Psi$. Then for $\left.z \in\right] 0, \kappa \varepsilon[$ and $\sigma \in \Gamma$

$$
\tilde{u}(\sigma, 0)=(\tilde{u}(\sigma, z))^{2}-2 \int_{0}^{z} \tilde{u}(\sigma, s) \frac{\partial \tilde{u}}{\partial z}(\sigma, s) d s .
$$

Integrating in $\sigma$ on $\Gamma$ we obtain that for all $z \in] 0 \kappa, \varepsilon[$,

$$
\|\tilde{u}(\cdot, 0)\|_{L^{2}(\Gamma)}^{2} \leq \int_{\Gamma}|\tilde{u}(\sigma, z)|^{2} d \sigma+C\|\tilde{u}\|_{L^{2}\left(\omega_{\varepsilon}\right)}\left\|\frac{\partial \tilde{u}}{\partial z}\right\|_{L^{2}\left(\omega_{\varepsilon}\right)} .
$$

Now we integrate this inequality in $z$ between 0 and $\kappa \varepsilon$ we obtain that :

$$
\varepsilon\|\tilde{u}(\cdot, 0)\|_{L^{2}(\Gamma)}^{2} \leq K\left(\|\tilde{u}\|_{L^{2}\left(\omega_{\varepsilon}\right)}^{2}+\varepsilon\|\tilde{u}\|_{L^{2}\left(\omega_{\varepsilon}\right)}\|\nabla \tilde{u}\|_{L^{2}\left(\omega_{\varepsilon}\right)}\right),
$$

that is dividing by $\varepsilon$,

$$
\|u\|_{L^{2}(\Gamma)} \leq K\left(\frac{1}{\sqrt{\varepsilon}}\|u\|_{L^{2}\left(\omega_{\varepsilon}\right)}+\|u\|_{L^{2}\left(\omega_{\varepsilon}\right)}^{\frac{1}{2}}\|\nabla u\|_{L^{2}\left(\omega_{\varepsilon}\right)}^{\frac{1}{2}}\right) .
$$

Proposition 2.2 We endow $H_{0}^{1}\left(\omega_{\varepsilon}\right)$ with the norm :

$$
\|u\|_{H_{0}^{1}\left(\omega_{\varepsilon}\right)}=\left(\int_{\omega_{\varepsilon}}\|\nabla u\|^{2}\right)^{\frac{1}{2}} .
$$

There exists $C$ such that for all $\varepsilon<\eta_{0}$, for all $u \in H_{0}^{1}\left(\omega_{\varepsilon}\right)$, then

$$
\|u\|_{L^{2}\left(\omega_{\varepsilon}\right)} \leq C \varepsilon\|u\|_{H_{0}^{1}\left(\omega_{\varepsilon}\right)}
$$

Proof : we prove this Poincaré inequality for regular $u$ as

$$
u(x)=\int_{0}^{\varphi(x)} \frac{\partial \tilde{u}}{\partial z}(P(x), s) d s
$$

and using Cauchy Schwarz inequality. 


\subsection{Divergence operator in thin domains}

The goal of this part is to prove the following fundamental result :

Theorem 2.1 There exists $\eta_{1}$ with $0<\eta_{1}<\eta_{0}$, there exists a constant $K$ such that for all $\varepsilon<\eta_{1}$, for all $g \in L^{2}\left(\omega_{\varepsilon}\right)$, if $\int_{\omega_{\varepsilon}} g=0$ there exists $U \in\left(H_{0}^{1}\left(\omega_{\varepsilon}\right)\right)^{3}$ such that :

$$
\left\{\begin{array}{l}
\operatorname{div} U=g \text { in } \omega_{\varepsilon} \\
\|U\|_{H_{0}^{1}\left(\omega_{\varepsilon}\right)} \leq \frac{K}{\varepsilon}\|g\|_{L^{2}\left(\omega_{\varepsilon}\right)}
\end{array}\right.
$$

Remark 2.2 It is well known that the divergence operator is a surjection from $\left(H_{0}^{1}\left(\omega_{\varepsilon}\right)\right)^{3}$ onto $L_{0}^{2}\left(\omega_{\varepsilon}\right)=\left\{g \in L^{2}\left(\omega_{\varepsilon}\right), \quad \int_{\omega_{\varepsilon}} g=0\right\}$, and so there exists a constant such that estimate (2.2) occurs. Here we determine the dependance of this constant on the thickness $\kappa \varepsilon$ of the thin domain.

Remark 2.3 For fixed domain, this proposition is proved in details in [10] (cf Theorem 2.1. p. 18). Here we adapt their proof for variable domains.

Proof of Theorem 2.1 :

If $U: \omega_{\varepsilon} \longrightarrow \mathbb{R}^{3}$ and $g: \omega_{\varepsilon} \longrightarrow \mathbb{R}^{3}$, we denote $\tilde{U}=u \circ \Psi$ and $\tilde{g}=g \circ \Psi$. Then equation (2.2) is equivalent to :

$$
\left.\frac{\partial \tilde{U}_{N}}{\partial s}+G_{s} \tilde{U}_{N}+\operatorname{div}_{\Gamma_{s}} \tilde{U}_{T}=\tilde{g} \text { for } s \in\right] 0, \kappa \varepsilon[\text { and } \sigma \in \Gamma .
$$

We seek $\tilde{U}$ on the form :

$$
\left\{\begin{array}{l}
\tilde{U}_{N}(\sigma, s)=\frac{\varepsilon}{\eta_{0}} Y_{N}\left(\sigma, \frac{\eta_{0} s}{\varepsilon}\right) \\
\tilde{U}_{T}(\sigma, s)=Y_{T}\left(\sigma, \frac{\eta_{0} s}{\varepsilon}\right)
\end{array}\right.
$$

where $Y$ is defined on $\Gamma \times] 0, \kappa \eta_{0}[$.

Setting $h(\sigma, s)=\tilde{g}\left(\sigma, \frac{\varepsilon s}{\eta_{0}}\right)$, the existence of $X$ is equivalent to the existence of $\left.Y: \Gamma \times\right] 0, \kappa \eta_{0}[$ satisfying :

$$
\left.\frac{\partial Y_{N}}{\partial z}+\frac{\varepsilon}{\eta_{0}} G_{\frac{\varepsilon z}{\eta_{0}}} Y_{N}+\operatorname{div}_{\Gamma_{\frac{\varepsilon z}{\eta_{0}}}} Y_{T}=h \text { for }(\sigma, z) \in \Gamma \times\right] 0, \kappa \eta_{0}[.
$$

We define $L^{2}$ and $H_{0}^{1}$ spaces on $\left.\Gamma \times\right] 0, \kappa \eta_{0}[$ :

$$
\begin{aligned}
& L^{2}(\Gamma \times] 0, \kappa \eta_{0}[)=\{v: \Gamma \times] 0, \kappa \eta_{0}\left[\longrightarrow \mathbb{R}, v \circ \Psi \in L^{2}\left(\omega_{\varepsilon}\right)\right\} \\
& H_{0}^{1}(\Gamma \times] 0, \kappa \eta_{0}[)=\{v: \Gamma \times] 0, \kappa \eta_{0}\left[\longrightarrow \mathbb{R}, v \circ \Psi \in H_{0}^{1}\left(\omega_{\varepsilon}\right)\right\}
\end{aligned}
$$

We endow $L^{2}(\Gamma \times] 0, \kappa \eta_{0}[)$ with a family of scalar products :

$$
<u \mid v>_{\varepsilon}=\int_{0}^{\kappa \eta_{0}} \int_{\Gamma} u(\sigma, z) v(\sigma, z) \gamma_{\frac{\varepsilon z}{\eta_{0}}}(\sigma) d z d \sigma
$$

and we denote \|\|$_{\varepsilon}$ the norm associated with this scalar product. 
We remark that the composition by $\Psi$ is an isometric map from $L^{2}\left(\omega_{\eta_{0}}\right)$ to $L^{2}(\Gamma \times] 0, \kappa \eta_{0}[)$ endowed with the scalar product $<\mid>_{\eta_{0}}$.

For $u: \Gamma \times] 0, \kappa \eta_{0}\left[\longrightarrow \mathbb{R}\right.$ we denote $\nabla_{\varepsilon}$ the following operator :

$$
\nabla_{\varepsilon} u(\sigma, z)=\frac{\partial u}{\partial z} n(\sigma)+\nabla_{\Gamma_{\frac{\varepsilon z}{\eta_{0}}}} u
$$

For $Y: \Gamma \times] 0, \kappa \eta\left[\longrightarrow \mathbb{R}^{3}\right.$ we consider $\operatorname{div}_{\varepsilon}$ the operator :

$$
\operatorname{div}_{\varepsilon} Y(\sigma, z)=\frac{\partial Y_{N}}{\partial z}+\frac{\varepsilon}{\eta_{0}} G_{\frac{\varepsilon z}{\eta_{0}}} Y_{N}+\operatorname{div}_{\Gamma_{\frac{\varepsilon z}{\eta_{0}}}} Y_{T}
$$

By construction $\operatorname{div}_{\varepsilon}$ and $\nabla_{\varepsilon}$ are in duality for the scalar product $\langle\mid\rangle_{\varepsilon}$.

Let $L_{0, \varepsilon}^{2}(\Gamma \times] 0, \kappa \eta_{0}[)$ defined by :

$$
L_{0, \varepsilon}^{2}(\Gamma \times] 0, \kappa \eta_{0}[)=\left\{h \in L^{2}(\Gamma \times] 0, \eta_{0}[), \quad \int_{0}^{\kappa \eta_{0}} \int_{\Gamma} h(\sigma, s) \gamma_{\frac{\varepsilon s}{\eta_{0}}}(\sigma) d s d \sigma=0\right\} .
$$

We have then the following proposition :

Proposition 2.3 There exists a constant $C$ such that for all $\varepsilon<\eta_{0}$, for all $h \in L_{0, \varepsilon}^{2}(\Gamma \times] 0, \kappa \eta_{0}[)$, there exists $V \in\left(H_{0}^{1}(\Gamma \times] 0, \kappa \eta_{0}[)\right)^{3}$ such that :

$$
\left\{\begin{array}{l}
\operatorname{div}_{\varepsilon} V=h \\
\left\|\nabla_{\varepsilon} V\right\|_{\varepsilon} \leq C\|h\|_{\varepsilon}
\end{array}\right.
$$

Assuming that Proposition 2.3 is true we can complete the proof of Proposition 2.1.

\section{End of the proof of Proposition 2.1}

Let $g \in L_{0}^{2}\left(\omega_{\varepsilon}\right)$. We define $\left.h: \Gamma \times\right] 0, \kappa \eta_{0}[$ by

$$
h(\sigma, z)=\tilde{g}\left(\sigma, \frac{\varepsilon z}{\eta_{0}}\right) .
$$

We remark that :

$$
\int_{0}^{\kappa \eta_{0}} \int_{\Gamma} h(\sigma, s) \gamma_{\frac{\varepsilon s}{\eta_{0}}}(\sigma) d s d \sigma=\frac{\eta_{0}}{\varepsilon} \int_{0}^{\kappa \varepsilon} \int_{\Gamma} h\left(\sigma, \frac{\eta_{0} z}{\varepsilon}\right) \gamma_{z}(\sigma) d z d \sigma=\int_{\omega_{\varepsilon}} g=0
$$

and so $h \in L_{0, \varepsilon}^{2}(\Gamma \times] 0, \kappa \eta_{0}[)$.

According to Proposition 2.3 there exists $V \in\left(H_{0}^{1}(\Gamma \times] 0, \kappa \eta_{0}[)\right)^{3}$ satisfying (2.4). We then define $U: \omega_{\varepsilon} \longrightarrow \mathbb{R}^{3}$ by :

$$
U_{N}(x)=\frac{\varepsilon}{\eta_{0}} V_{N}\left(P(x), \frac{\eta_{0}}{\varepsilon} \varphi(x)\right) \quad \text { and } U_{T}(x)=V_{T}\left(P(x), \frac{\eta_{0}}{\varepsilon} \varphi(x)\right)
$$

and we already know that

$$
\operatorname{div} U=g \text { in } \omega_{\varepsilon}
$$


Furthermore,

$$
\begin{aligned}
\|\nabla U\|_{L^{2}\left(\omega_{\varepsilon}\right)}^{2} & =\int_{0}^{\kappa \varepsilon} \int_{\Gamma}\left(\left|\frac{\partial \tilde{U}}{\partial s}\right|^{2}+\left|\nabla_{\Gamma_{s}} \tilde{U}\right|^{2}\right) \gamma_{s}(\sigma, s) d s d \sigma \\
& =\int_{0}^{\kappa \varepsilon} \int_{\Gamma}\left(\left|\nabla_{\Gamma_{s}} Y_{T}\right|^{2}+\frac{\eta_{0}^{2}}{\varepsilon^{2}}\left|\frac{\partial Y_{T}}{\partial z}\right|^{2}+\frac{\varepsilon^{2}}{\eta_{0}^{2}}\left|\nabla_{\Gamma_{s}} Y_{N}\right|^{2}+\left|\frac{\partial Y_{N}}{\partial z}\right|^{2}\right)\left(\sigma, \frac{\eta_{0} s}{\varepsilon}\right) \gamma_{s}(\sigma, s) d s d \sigma \\
& \leq \frac{\eta_{0}^{2}}{\varepsilon^{2}} \int_{0}^{\kappa \varepsilon} \int_{\Gamma}\left(\left|\nabla_{\Gamma_{s}} Y_{T}\right|^{2}+\left|\frac{\partial Y_{T}}{\partial z}\right|^{2}+\left|\nabla_{\Gamma_{s}} Y_{N}\right|^{2}+\left|\frac{\partial Y_{N}}{\partial z}\right|^{2}\right)\left(\sigma, \frac{\eta_{0} s}{\varepsilon}\right) \gamma_{s}(\sigma, s) d s d \sigma \\
& \leq \frac{\eta_{0}}{\varepsilon} \int_{0}^{\kappa \eta_{0}} \int_{\Gamma}\left(\left|\nabla_{\Gamma_{\frac{\varepsilon z}{\eta_{0}}}} Y_{T}\right|^{2}+\left|\frac{\partial Y_{T}}{\partial z}\right|^{2}+\left|\nabla_{\Gamma_{\frac{\varepsilon z}{\eta_{0}}}} Y_{N}\right|^{2}+\left|\frac{\partial Y_{N}}{\partial z}\right|^{2}\right)(\sigma, z) \gamma_{\frac{\varepsilon z}{\eta_{0}}}(\sigma) d z d \sigma \\
& \leq \frac{\eta_{0}}{\varepsilon}\left\|\nabla_{\varepsilon} Y\right\|_{\varepsilon}^{2} \\
& \leq C \frac{\eta_{0}}{\varepsilon}\|h\|_{\varepsilon}^{2} \\
& \leq C \frac{\eta_{0}^{2}}{\varepsilon^{2}}\|g\|_{L^{2}\left(\omega_{\varepsilon}\right)}^{2}
\end{aligned}
$$

Hence this complete the proof of Proposition 2.1.

\section{Proof of Proposition 2.3}

We endow $H_{0}^{1}(\Gamma \times] 0, \kappa \eta_{0}[)$ with the family of norms :

$$
\|u\|_{1, \varepsilon}=\left\|\nabla_{\varepsilon} u\right\|_{\varepsilon} .
$$

We remark that the composition by $\Psi$ is an isometric map from $H_{0}^{1}\left(\omega_{\eta_{0}}\right)$ to $H_{0}^{1}(\Gamma \times] 0, \kappa \eta_{0}[)$ endowed with the norm $\|\cdot\|_{1, \eta_{0}}$.

The dual space $H^{-1}$ can be endowed with the family of norms :

$$
\forall l \in L^{2}, \quad\|l\|_{-1, \varepsilon}=\sup _{u \in H_{0}^{1}(\Gamma \times] 0, \kappa \eta_{0}[)} \frac{|<l| u>_{\varepsilon} \mid}{\|u\|_{1, \varepsilon}} .
$$

Lemma 2.1 There exists $C_{1}$ and $C_{2}$ such that for all $\left.\varepsilon \in\right] 0, \eta_{0}[$,

$$
\begin{aligned}
& \text { (1) } \forall u \in L^{2}(\Gamma \times] 0, \kappa \eta_{0}[), \quad C_{1}\|u\|_{\eta_{0}} \leq\|u\|_{\varepsilon} \leq C_{2}\|u\|_{\eta_{0}} \\
& \text { (2) } \forall u \in H_{0}^{1}(\Gamma \times] 0, \kappa \eta_{0}[), \quad C_{1}\left\|\nabla_{\eta_{0}} u\right\|_{\eta_{0}} \leq\left\|\nabla_{\varepsilon}\right\|_{\eta_{0}} \leq C_{2}\left\|\nabla_{\eta_{0}} u\right\|_{\eta_{0}} \\
& \text { (3) } \forall l \in H^{-1}(\Gamma \times] 0, \kappa \eta_{0}[), \quad C_{1}\|l\|_{-1, \eta_{0}} \leq\|l\|_{-1, \varepsilon} \leq C_{2}\|l\|_{-1, \eta_{0}}
\end{aligned}
$$

Proof of Lemma 2.1.

We remark that $(\sigma, z) \mapsto \gamma_{s}(\sigma)$ is regular, $\gamma_{0} \equiv 1, \Gamma$ is compact, thus, even if it means reducing $\eta_{0}$, we can suppose that for all $z \in\left[0, \kappa \eta_{0}\right]$, for all $\sigma \in \Gamma$,

$$
\frac{9}{10} \leq \gamma_{z}(\sigma) \leq \frac{11}{10}
$$


Therefore for all $z \in\left[0, \kappa \eta_{0}\right]$, for all $\varepsilon \in\left[0, \eta_{0}\right]$, for all $\sigma \in \Gamma$,

$$
\frac{9}{11} \gamma_{z}(\sigma) \leq \gamma_{\frac{\varepsilon z}{\eta_{0}}}(\sigma) \leq \frac{11}{9} \gamma_{z}(\sigma)
$$

For $u \in L^{2}(\Gamma \times] 0, \kappa \eta_{0}[)$,

$$
\|u\|_{\varepsilon}^{2}=\int_{0}^{\kappa \eta_{0}} \int_{\Gamma} u^{2}(\sigma, z) \gamma_{\frac{\varepsilon z}{\eta_{0}}} d z d \sigma
$$

thus

$$
\frac{9}{11}\|u\|_{\eta_{0}}^{2} \leq\|u\|_{\varepsilon}^{2} \leq \frac{11}{9}\|u\|_{\eta_{0}}^{2}
$$

For the second inequality,

$$
\left\|\nabla_{\varepsilon} u\right\|_{\eta_{0}}^{2}=\left\|M_{\varepsilon}(s, \sigma) \nabla_{\eta_{0}} u\right\|_{\eta_{0}}
$$

where $M_{\varepsilon}(s, \sigma)$ is the endomorphism defined by :

$$
M_{\varepsilon}(s, \sigma)(\xi)=\xi_{N}+\left(I d+\frac{\varepsilon s}{\eta_{0}} d n(\sigma)\right)^{-1} \circ(I d+s d n(\sigma))\left(\xi_{T}\right)
$$

(since $\left.\nabla_{\Gamma_{s}}=(I d+s d n(\sigma)) \nabla_{\Gamma_{0}}\right)$.

Now for $s=0, M_{\varepsilon}(0, \sigma)=I d$ so even if it means reducing $\eta_{0}$, since $(\sigma, \varepsilon, \sigma) \mapsto M_{\varepsilon}(s, \sigma)$ is regular, we can suppose that :

$$
\left|M_{\varepsilon}(\sigma, s)\right| \leq K \quad \text { and }\left|\left(M_{\varepsilon}(\sigma, s)\right)^{-1}\right| \leq K .
$$

Hence we have :

$$
\frac{1}{K}\left\|\nabla_{\eta_{0}} u\right\|_{\eta_{0}}^{2} \leq\left\|\nabla_{\varepsilon} u\right\|_{\eta_{0}}^{2} \leq K\left\|\nabla_{\eta_{0}} u\right\|_{\eta_{0}}^{2} .
$$

In conclusion, for the last inequality, we remark that for $l \in L^{2}(\Gamma \times] 0, \kappa \eta_{0}[)$, for $\xi \in H_{0}^{1}(\Gamma \times] 0, \kappa \eta_{0}[)$, we have :

$$
\begin{aligned}
<l \mid \xi>_{\varepsilon} & =\int_{0}^{\kappa \eta_{0}} \int_{\Gamma} l(\sigma, z) \xi(\sigma, z) \gamma_{\frac{\varepsilon z}{\eta_{0}}}(\sigma) d z d \sigma \\
& =\int_{0}^{\kappa \eta_{0}} \int_{\Gamma} l(\sigma, z) \xi(\sigma, z) \frac{\gamma_{\frac{\varepsilon z}{\eta_{0}}}(\sigma)}{\gamma_{z}(\sigma)} \gamma_{z}(\sigma) d z d \sigma
\end{aligned}
$$

hence

$$
|<l| \xi>_{\varepsilon} \mid \leq\|l\|_{-1, \eta_{0}}\left\|\xi \frac{\gamma_{\frac{\varepsilon z}{\eta_{0}}}}{\gamma_{z}}\right\|_{1, \eta_{0}}
$$

Now,

$$
\left\|\xi \frac{\gamma_{\frac{\varepsilon z}{\eta_{0}}}}{\gamma_{z}}\right\|_{1, \eta_{0}} \leq C\|\xi\|_{1, \eta_{0}}\left(\left\|\frac{\gamma_{\frac{\varepsilon z}{\eta_{0}}}}{\gamma_{z}}\right\|_{L^{\infty}}+\left\|\nabla_{\eta_{0}}\left(\frac{\gamma_{\frac{\varepsilon z}{\eta_{0}}}}{\gamma_{z}}\right)\right\|_{L^{\infty}}\right)
$$

and since $(\sigma, s) \mapsto \gamma_{s}(\sigma)$ is regular, there exists an universal constant $K$ such that for all $\varepsilon \in\left[0, \eta_{0}\right]$

$$
\left(\left\|\frac{\gamma_{\frac{\varepsilon z}{\eta_{0}}}}{\gamma_{z}}\right\|_{L^{\infty}}+\left\|\nabla_{\eta_{0}}\left(\frac{\gamma \frac{\varepsilon z}{\eta_{0}}}{\gamma_{z}}\right)\right\|_{L^{\infty}}\right) \leq C
$$

Hence we have

$$
\begin{aligned}
|<l| \xi>_{\varepsilon} \mid & \leq C\|l\|_{-1, \eta_{0}}\|\nabla \xi\|_{\eta_{0}} \\
& \leq C\|l\|_{-1, \eta_{0}} K\left\|\nabla_{\varepsilon} \xi\right\|_{\eta_{0}} \\
& \leq C K \sqrt{\frac{11}{10}}\|l\|_{-1, \eta_{0}}\left\|\nabla_{\varepsilon} \xi\right\|_{\varepsilon} .
\end{aligned}
$$


Hence we obtain that :

$$
\|l\|_{-1, \varepsilon} \leq C K \sqrt{\frac{11}{10}}\|l\|_{-1, \eta_{0}}
$$

and we can obtain in the same way the inverse inequality.

Lemma 2.2 There exists a constant $K$ such that for all $\varepsilon \in\left[0, \eta_{0}\right]$, for all $u \in L^{2}(\Gamma \times] 0, \kappa \eta_{0}[)$,

$$
\frac{1}{K}\left\|\nabla_{\eta_{0}} u\right\|_{-1, \eta_{0}} \leq\left\|\nabla_{\varepsilon} u\right\|_{-1, \eta_{0}} \leq K\left\|\nabla_{\eta_{0}} u\right\|_{-1, \eta_{0}} .
$$

Proof of Lemma 2.2. We prove the inequality for $u$ regular and we conclude the proof by density. For $\xi \in H_{0}^{1}(\Gamma \times] 0, \kappa \eta_{0}[)$ we have :

$$
\begin{aligned}
<\nabla_{\varepsilon} u \mid \xi>_{\eta_{0}} & =\int_{0}^{\kappa \eta_{0}} \int_{\Gamma}\left(M_{\varepsilon}(s, \sigma) \nabla_{\eta_{0}} u \cdot \xi\right) \gamma_{s} \\
& =\int_{0}^{\kappa \eta_{0}} \int_{\Gamma}\left(\nabla_{\eta_{0}} u \cdot M_{\varepsilon}(s, \sigma) \xi\right) \gamma_{s}
\end{aligned}
$$

hence

$$
\begin{aligned}
\left|<\nabla_{\varepsilon} u\right| \xi>_{\eta_{0}} \mid & \leq\left\|\nabla_{\eta_{0}} u\right\|_{-1, \eta_{0}}\left\|M_{\varepsilon}(s, \sigma) \xi\right\|_{1, \eta_{0}} \\
& \leq\left\|\nabla_{\eta_{0}} u\right\|_{-1, \eta_{0}}\left(\left\|M_{\varepsilon}(s, \sigma)\right\|_{L^{\infty}}+\left\|\nabla_{\eta_{0}} M_{\varepsilon}(s, \sigma)\right\|_{L^{\infty}}\right)\|\xi\|_{1, \eta_{0}}
\end{aligned}
$$

thus since $M_{\varepsilon}$ and $\nabla_{\eta_{0}} M_{\varepsilon}$ are uniformly bounded, there exists $K$ such that

$$
\left\|\nabla_{\varepsilon} u\right\|_{-1, \eta_{0}} \leq K\left\|\nabla_{\eta_{0}} u\right\|_{-1, \eta_{0}} .
$$

In the same way, we obtain the inverse inequality.

Lemma 2.3 There exists $C$ there exists $\eta_{1}<\eta_{0}$ such that for all $\varepsilon<\eta_{1}$, for all $u \in L_{0, \varepsilon}^{2}(\Gamma \times] 0, \kappa \eta_{0}[)$,

$$
\|u\|_{\varepsilon} \leq C\left\|\nabla_{\varepsilon} u\right\|_{-1, \varepsilon} .
$$

Proof of Lemma 2.3. We suppose that Lemma 2.3 is false. Then there exists a subsequence $\varepsilon_{n} \longrightarrow 0$, and $u_{n} \in L_{0, \varepsilon_{n}}^{2}(\Gamma \times] 0, \kappa \eta_{0}[)$ such that :

$$
\left\|u_{n}\right\|_{\varepsilon_{n}}=1 \text { and }\left\|\nabla_{\varepsilon_{n}} u_{n}\right\|_{-1, \varepsilon_{n}} \leq \frac{1}{n} .
$$

With (1) in (2.5) we obtain that :

$$
\forall n, \frac{1}{C_{2}} \leq\left\|u_{n}\right\|_{\eta_{0}} \leq \frac{1}{C_{2}}
$$

thus we can extract a subsequence still denoted $u_{n}$ such that :

$$
u_{n} \rightarrow u \text { in } L^{2}(\Gamma \times] 0, \kappa \eta_{0}[) \text { weak. }
$$

We remark that for all $n$, with Lemma 2.1 and Lemma 2.2,

$$
\begin{aligned}
\left\|\nabla_{\eta_{0}} u_{n}\right\|_{-1, \eta_{0}} & \leq K\left\|\nabla_{\varepsilon_{n}} u_{n}\right\|_{-1, \eta_{0}} \\
& \leq K C\left\|\nabla_{\varepsilon_{n}} u_{n}\right\|_{-1, \varepsilon_{n}} \\
& \leq \frac{K C}{n} .
\end{aligned}
$$


Thus $\nabla_{\eta_{0}} u_{n}$ tends to zero in $H^{-1}(\Gamma \times] 0, \kappa \eta_{0}[)$ strongly, hence $u$ is constant.

Now, from [10], we know that there exists $C$ such that :

$$
\forall v \in L^{2}\left(\omega_{\eta_{0}}\right), \quad\|v\|_{L^{2}\left(\omega_{\eta_{0}}\right)} \leq C\left(\|v\|_{H^{-1}\left(\omega_{\eta_{0}}\right)}+\|\nabla v\|_{H^{-1}\left(\omega_{\eta_{0}}\right)}\right) .
$$

Since the composition with $\Psi$ is an isometry, we obtain that:

$$
\forall u \in L^{2}(\Gamma \times] 0, \kappa \eta_{0}[), \quad\|u\|_{\eta_{0}} \leq C\left(\|u\|_{-1, \eta_{0}}+\left\|\nabla_{\eta_{0}} u\right\|_{-1, \eta_{0}}\right) .
$$

Using this inequality, since the injection of $L^{2}$ in $H^{-1}$ is compact, the Cauchy criterium in $L^{2}(\Gamma \times] 0, \eta_{0}[)$ gives that :

$$
u_{n} \longrightarrow u \text { strongly in } L^{2}(\Gamma \times] 0, \kappa \eta_{0}[)
$$

Thus with (2.6) we have :

$$
\|u\|_{\eta_{0}} \geq \frac{1}{C_{2}}
$$

On the other hand, we know that for all $n$ we have :

$$
\int_{0}^{\kappa \eta_{0}} \int_{\Gamma} u_{n}(\sigma, z) \gamma_{\frac{\varepsilon_{n} z}{\eta_{0}}}=0 .
$$

We know that $u_{n}$ tends to $u$ in $L^{2}$ strongly and that $\frac{\varepsilon_{n} z}{\eta_{0}}$ tends to 1 uniformly. Then, we have :

$$
\int_{0}^{\kappa \eta_{0}} \int_{\Gamma} u=0
$$

Since $u$ is a constant, this implies that $u \equiv 0$, and this leads to a contradiction with 2.7 and concludes the proof of Lemma 2.3.

\section{End of the proof of Proposition 2.3.}

Following [10], using their Theorem 2.1 page 18 and a duality argument in the spirit of Corollary 2.4 page 24, we conclude the proof of Proposition 2.3.

\section{BKW Method for the penalized thin layer problem}

\subsection{Formal asymptotic expansion}

We consider the following penalized problem :

$$
\left\{\begin{array}{lr}
\frac{\partial u^{\varepsilon}}{\partial t}-\Delta u^{\varepsilon}+\left(u^{\varepsilon} \cdot \nabla\right) u^{\varepsilon}+\nabla \pi^{\varepsilon}+\frac{1}{\varepsilon} \chi_{\omega_{\varepsilon}} u^{\varepsilon}=f & \text { in } \mathbb{R}^{+} \times \mathcal{U}^{\varepsilon} \\
\operatorname{div} u^{\varepsilon}=0 & \text { in } \mathbb{R}^{+} \times \mathcal{U}^{\varepsilon}, \\
u^{\varepsilon}=0 & \text { on } \mathbb{R}^{+} \times \partial \Omega, \\
u^{\varepsilon}=0 & \text { on } \mathbb{R}^{+} \times \Gamma_{\varepsilon} \\
u^{\varepsilon}(0, x)=u_{0}^{\varepsilon}(x) & \text { in } \mathcal{U}^{\varepsilon} .
\end{array}\right.
$$

We denote by $v^{\varepsilon}\left(\operatorname{resp} . p^{\varepsilon}\right)$ the restriction of $u^{\varepsilon}\left(\operatorname{resp} . \pi^{\varepsilon}\right)$ in $\mathcal{U}=\Omega \backslash \bar{\omega}$ and $w^{\varepsilon}$ (resp. $q^{\varepsilon}$ ) the restriction of $u^{\varepsilon}\left(\right.$ resp. $\left.\pi^{\varepsilon}\right)$ on $\omega$. 
Equation (3.1) is equivalent to the following system on $v^{\varepsilon}$ and $w^{\varepsilon}$ :

$$
\begin{cases}\frac{\partial v^{\varepsilon}}{\partial t}-\Delta v^{\varepsilon}+\left(v^{\varepsilon} \cdot \nabla\right) v^{\varepsilon}+\nabla p^{\varepsilon}=f & \text { in } \mathbb{R}_{t}^{+} \times \mathcal{U} \\ \frac{\partial w^{\varepsilon}}{\partial t}-\Delta w^{\varepsilon}+\left(w^{\varepsilon} \cdot \nabla\right) w^{\varepsilon}+\nabla q^{\varepsilon}+\frac{1}{\varepsilon} w^{\varepsilon}=0 & \text { in } \mathbb{R}_{t}^{+} \times \omega_{\varepsilon} \\ \operatorname{div} v^{\varepsilon}=0 & \text { in } \mathbb{R}_{t}^{+} \times \mathcal{U} \\ \operatorname{div} w^{\varepsilon}=0 & \text { in } \mathbb{R}_{t}^{+} \times \omega_{\varepsilon} \\ w^{\varepsilon}=0 & \text { in } \mathbb{R}_{t}^{+} \times \Gamma_{\varepsilon} \\ v^{\varepsilon}=w^{\varepsilon} & \text { on } \mathbb{R}_{t}^{+} \times \partial \omega \\ -\frac{\partial v^{\varepsilon}}{\partial n}+p^{\varepsilon} n=-\frac{\partial w^{\varepsilon}}{\partial n}+q^{\varepsilon} n & \text { on } \mathbb{R}_{t}^{+} \times \partial \omega \\ v^{\varepsilon}=0 & \text { on } \mathbb{R}_{t}^{+} \times \partial \Omega\end{cases}
$$

where $n$ is the outward unitary normal at $\partial \mathcal{U}$.

Remark 3.1 The boundary condition (6) in the previous equation comes from the variational formulation of Equation (3.1) since :

$$
\int_{\mathcal{U}}\left(-\Delta u^{\varepsilon}+\nabla \pi^{\varepsilon}\right) \cdot \psi=\int_{\mathcal{U}}\left(\left(\nabla u^{\varepsilon} \cdot \nabla \psi\right)-\pi^{\varepsilon} \operatorname{div} \psi\right)+\int_{\Gamma}\left(-\frac{\partial u^{\varepsilon}}{\partial n}+\pi^{\varepsilon} n\right) \cdot \psi
$$

and

$$
\int_{\omega_{\varepsilon}}\left(-\Delta u^{\varepsilon}+\nabla \pi^{\varepsilon}\right) \cdot \psi=\int_{\omega_{\varepsilon}}\left(\left(\nabla u^{\varepsilon} \cdot \nabla \psi\right)-\pi^{\varepsilon} \operatorname{div} \psi\right)-\int_{\Gamma}\left(-\frac{\partial u^{\varepsilon}}{\partial n}+\pi^{\varepsilon} n\right) \cdot \psi
$$

since $n$ is outing from $\mathcal{U}$ and entering into $\omega_{\varepsilon}$.

We perform an asymptotic expansion of $v^{\varepsilon}, w^{\varepsilon}, p^{\varepsilon}$ and $q^{\varepsilon}$ of the form :

$$
\begin{aligned}
& v^{\varepsilon}(t, x)=V^{0}(t, x)+\varepsilon V^{1}(t, x)+\ldots, \\
& p^{\varepsilon}(t, x)=p^{0}(t, x)+\varepsilon p^{1}(t, x)+\ldots \\
& w^{\varepsilon}(t, x)=W^{0}\left(t, P(x), \frac{\varphi(x)}{\varepsilon}\right)+\varepsilon W^{1}\left(t, P(x), \frac{\varphi(x)}{\varepsilon}\right)+\ldots, \\
& q^{\varepsilon}(t, x)=q^{0}\left(t, P(x), \frac{\varphi(x)}{\varepsilon}\right)+\varepsilon q^{1}\left(t, P(x), \frac{\varphi(x)}{\varepsilon}\right)+\varepsilon^{2} q^{2}\left(t, P(x), \frac{\varphi(x)}{\varepsilon}\right)+\ldots
\end{aligned}
$$

where $P(x)$ is the projection of $x$ on $\Gamma$ and $\varphi(x)=\operatorname{dist}(x, \Gamma)$.

We assume that the terms $W^{i}: \mathbb{R}^{+} \times \Gamma \times[0, \kappa] \longrightarrow \mathbb{R}^{3}$

$$
\forall t \in \mathbb{R}^{+}, \forall \sigma \in \Gamma, \quad W^{i}(t, \sigma, z=\kappa)=0 .
$$

The coordinates of $\mathbb{R}^{+} \times \Gamma \times[0, \kappa]$ are denoted $(t, \sigma, z)$. 
We denote $W_{T}^{i}$ the tangential part of $W^{i}$, and $W_{N}^{i}$ its normal part :

$$
\begin{aligned}
& W_{N}^{i}(t, \sigma, z)=W^{i}(t, \sigma, z) \cdot n(\sigma) \\
& W_{T}^{i}(t, \sigma, z)=W^{i}(t, \sigma, z)-W_{N}^{i}(t, \sigma, z) n(\sigma)
\end{aligned}
$$

where $n(\sigma)$ is the outward unitary normal at $\partial \mathcal{U}$ at the point $\sigma$.

In (3.2) we will formaly replace $u^{\varepsilon}, v^{\varepsilon}, p^{\varepsilon}$ and $w^{\varepsilon}$ by their asymptotic expansion and we will identify the different powers of $\varepsilon$.

We recall that if $\tilde{\alpha}: \Gamma \times[0, \kappa] \longrightarrow \mathbb{R}$, if we denote $\alpha(x)=\tilde{\alpha}\left(P(x), \frac{\varphi(x)}{\varepsilon}\right)$, then

$$
\nabla \alpha(x)=\frac{1}{\varepsilon} \frac{\partial \tilde{\alpha}}{\partial z}\left(P(x), \frac{\varphi(x)}{\varepsilon}\right) n(P(x))+\nabla_{\Gamma_{\varphi(x)}} \tilde{\alpha}\left(P(x), \frac{\varphi(x)}{\varepsilon}\right)
$$

and

$$
\Delta \alpha(x)=\frac{1}{\varepsilon^{2}} \frac{\partial^{2} \tilde{\alpha}}{\partial z^{2}}\left(P(x), \frac{\varphi(x)}{\varepsilon}\right)+\frac{1}{\varepsilon} G_{\varphi(x)}(P(x)) \frac{\partial \tilde{\alpha}}{\partial z}\left(P(x), \frac{\varphi(x)}{\varepsilon}\right)+\Delta_{\Gamma_{\varphi(x)}} \tilde{\alpha}\left(P(x), \frac{\varphi(x)}{\varepsilon}\right) .
$$

Furthermore, if $\tilde{\beta}: \Gamma \times[0, \kappa] \longrightarrow \mathbb{R}^{3}$, if $\beta(x)=\tilde{\beta}\left(P(x), \frac{\varphi(x)}{\varepsilon}\right)$, then

$$
\operatorname{div} \beta(x)=\frac{1}{\varepsilon} \frac{\partial \tilde{\beta}_{N}}{\partial z}\left(P(x), \frac{\varphi(x)}{\varepsilon}\right)+G_{\varphi(x)}(P(x)) \tilde{\beta}_{N}\left(P(x), \frac{\varphi(x)}{\varepsilon}\right)+\operatorname{div}_{\Gamma_{\varphi(x)}} \tilde{\beta}_{T}\left(P(x), \frac{\varphi(x)}{\varepsilon}\right) .
$$

In addition we can perform an asymptotic expansion of $G_{s}, \nabla_{\Gamma_{s}}$ and $\operatorname{div} \Gamma_{s}$ and we have :

- $G_{\varepsilon z}(\sigma)=G_{0}(\sigma)+\varepsilon z G_{0}^{\prime}(\sigma)+\mathcal{O}\left(\varepsilon^{2}\right)$,

- for $\tilde{Z}: \Gamma \longrightarrow T \Gamma$ a tangential vector field,

$$
\operatorname{div}_{\Gamma_{\varepsilon z}} \tilde{Z}(\sigma)=\operatorname{div}_{\Gamma} \tilde{Z}+\varepsilon z \operatorname{div}_{\Gamma}^{\prime} \tilde{Z}+\mathcal{O}\left(\varepsilon^{2}\right)
$$

with

$$
\operatorname{div}_{\Gamma}^{\prime} \tilde{Z}=-G_{0}(\sigma) \operatorname{div}_{\Gamma} \tilde{Z}+\operatorname{div}_{\Gamma}\left(G_{0}(\sigma) \tilde{Z}\right)-\operatorname{div}_{\Gamma}(d n(\sigma) \tilde{Z})
$$

- for $\tilde{\alpha}: \Gamma \longrightarrow \mathbb{R}$,

$$
\nabla_{\Gamma_{\varepsilon z}} \tilde{\alpha}(\sigma)=\nabla_{\Gamma} \tilde{\alpha}(\sigma)+\varepsilon z \nabla_{\Gamma}^{\prime} \tilde{\alpha}(\sigma)+\mathcal{O}\left(\varepsilon^{2}\right)
$$

with $\nabla_{\Gamma}^{\prime} \tilde{\alpha}(\sigma)=-d n(\sigma)\left(\nabla_{\Gamma} \tilde{\alpha}(\sigma)\right)$

Remark 3.2 Since $n(\sigma)$ does not depend on $z$, we remark that $\left(\frac{\partial \tilde{\beta}}{\partial z}\right)_{N}=\left(\frac{\partial \tilde{\beta}}{\partial z} \cdot n\right)=$ $\frac{\partial}{\partial z}(\tilde{\beta} \cdot n)=\frac{\partial \tilde{\beta}_{N}}{\partial z}$ and so $\left(\frac{\partial \tilde{\beta}}{\partial z}\right)_{T}=\frac{\partial \tilde{\beta}_{T}}{\partial z}$. 
Step 1: we write (2) at order $\varepsilon^{-2}$ :

$$
-\frac{\partial^{2} W^{0}}{\partial z^{2}}=0
$$

With (7) at order $\varepsilon^{-1}$, on $\Gamma$, i.e. for $z=0, W_{z}^{0}=0$. Since $W^{0}(\sigma, z=\kappa)=0$, we obtain :

$$
W^{0} \equiv 0 \text {. }
$$

Step 2 : $\quad$ we write (1), (3) and (6) at order $\varepsilon^{0}$ and we obtain :

$$
\left\{\begin{array}{l}
\frac{\partial V^{0}}{\partial t}-\Delta V^{0}+\left(V^{0} \cdot \nabla\right) V^{0}+\nabla p^{0}=f \quad \text { in } \mathbb{R}^{+} \times \mathcal{U} \\
\operatorname{div} V^{0}=0 \quad \text { in } \mathbb{R}^{+} \times \mathcal{U} \\
V^{0}=W^{0}=0 \quad \text { on } \mathbb{R}^{+} \times \partial \mathcal{U}
\end{array}\right.
$$

which determine $V^{0}$ completely (if we precise the initial data).

Remark 3.3 We will prove in the following section that since $V^{0}$ is regular in $\mathcal{U}$, since $\operatorname{div} V^{0}=$ 0 in $\mathcal{U}$ and as $V^{0}=0$ on $\Gamma$, we have :

$$
\left(\frac{\partial V^{0}}{\partial n}\right)_{N}=0 \text { on } \Gamma
$$

that is $\frac{\partial V^{0}}{\partial n}$ is tangential on the boundary $\Gamma$.

Step 3 : with (4) at order $\varepsilon^{0}$ we obtain $\frac{\partial W_{N}^{1}}{\partial z}=0$. Hence $W_{N}^{1}$ does not depend on $z$ and since it is zero at $z=1$,

$$
W_{N}^{1} \equiv 0 .
$$

(2) at order $\varepsilon^{-1}$ gives :

$$
-\frac{\partial^{2} W^{1}}{\partial z^{2}}+W^{0}+\frac{\partial q^{0}}{\partial z} n=0
$$

and taking the scalar product of this equation with $n$ we obtain that $q^{0}$ does not depend on $z$ (since $W^{0}=0$ ).

With (7) at order $\varepsilon^{0}$ we have :

$$
-\frac{\partial W^{1}}{\partial z}+q^{0} n=p^{0} n-\frac{\partial V^{0}}{\partial n} \text { on } \Gamma .
$$

Taking this equation scalar $n$ we have :

$$
q^{0}(z=0)=p^{0}
$$

and we extend this expression in $\Gamma \times[0,1]$ to obtain $q^{0}$ :

$$
q^{0}(\sigma, z)=p^{0}(\sigma)
$$


Remark 3.4 We obtain here that the pressure in continuous at the boundary $\Gamma$.

We know then that

$$
\left\{\begin{array}{l}
-\frac{\partial^{2} W^{1}}{\partial z^{2}}=0 \\
W^{1}(z=\kappa)=0 \\
\frac{\partial W^{1}}{\partial z}(z=0)=\frac{\partial V^{0}}{\partial n}
\end{array}\right.
$$

that is

$$
W^{1}(t, \sigma, z)=(z-\kappa) \frac{\partial V^{0}}{\partial n}(t, \sigma)
$$

Step 4 : With (1), (3) and (6) at order 1 we obtain that

$$
\left\{\begin{array}{l}
\frac{\partial V^{1}}{\partial t}-\Delta V^{1}+V^{0} \cdot \nabla V^{1}+V^{1} \cdot \nabla V^{0}+\nabla p^{1}=0 \quad \text { in } \mathbb{R}^{+} \times \mathcal{U}, \\
\operatorname{div} V^{1}=0 \quad \text { in } \mathbb{R}^{+} \times \mathcal{U}, \\
V^{1}(t, x)=W^{1}(t, x, 0)=-\kappa \frac{\partial V^{0}}{\partial n} \quad \text { on } \mathbb{R}^{+} \times \Gamma,
\end{array}\right.
$$

hence we determine $V^{1}$.

Remark 3.5 The existence of a vector field satisfying $\operatorname{div} V^{1}=0$ in $\mathcal{U}, V^{1}=0$ on $\partial \Omega$ and $V^{1}=W^{1}$ on $\Gamma$ is assured by the fact that $\int_{\Gamma}\left(W^{1} \cdot n\right)=0\left(\right.$ since $\left.W_{N}^{1}=0\right)$.

Step 5 : (4) at order $\varepsilon^{0}$ gives :

$$
\frac{\partial W_{N}^{2}}{\partial z}+\operatorname{div}_{\Gamma_{0}} W_{T}^{1}=0
$$

thus the normal part of $W^{2}$ is given by :

$$
W_{N}^{2}(t, \sigma, z)=-\int_{\kappa}^{z} \operatorname{div}_{\Gamma_{0}} W_{T}^{1}(t, \sigma, \xi) d \xi=-\frac{1}{2}(z-\kappa)^{2} \operatorname{div}_{\Gamma}\left(\frac{\partial V^{0}}{\partial n}(t, \sigma)\right)
$$

We write (7) at order $\varepsilon$ and we obtain :

$$
-\frac{\partial W^{2}}{\partial z}+q^{1} n=p^{1} n-\frac{\partial V^{1}}{\partial n} \text { at } z=0 .
$$

Taking the scalar product of this equation with $n$ we determine $q^{1}$ at $z=0$ :

$$
q^{1}(t, \sigma, 0)=\left(p^{1}-\left(\frac{\partial V^{1}}{\partial n} \cdot n\right)\right)(t, \sigma)+\left(\frac{\partial W_{N}^{2}}{\partial z}\right)(t, \sigma, z=0)
$$


that is

$$
q^{1}(t, \sigma, 0)=\left(p^{1}-\left(\frac{\partial V^{1}}{\partial n} \cdot n\right)\right)(t, \sigma)+\kappa \operatorname{div}_{\Gamma}\left(\frac{\partial V^{0}}{\partial n}\right)
$$

With (2) at order $\varepsilon^{0}$ we have :

$$
-\frac{\partial^{2} W^{2}}{\partial z^{2}}-G_{0} \frac{\partial W^{1}}{\partial z}+\nabla_{\Gamma_{0}} q^{0}+\frac{\partial q^{1}}{\partial z} n+W^{1}=0
$$

Taking the normal part of this equation, since $W^{1}$ is tangential, we determine $\frac{\partial q^{1}}{\partial z}$

$$
\frac{\partial q^{1}}{\partial z}=\frac{\partial^{2} W_{N}^{2}}{\partial z^{2}}
$$

and then

$$
q^{1}(t, \sigma, z)=\left(p^{1}-\left(\frac{\partial V^{1}}{\partial n} \cdot n\right)\right)(t, \sigma)-(z-\kappa) \operatorname{div}_{\Gamma}\left(\frac{\partial V^{0}}{\partial n}\right)
$$

In order to precise the tangential part of $W^{2}$ we take the tangential part of (3.5) and the tangential part of (3.3) and we derive the equation satisfied by $W_{T}^{2}$ :

$$
\left\{\begin{array}{l}
\frac{\partial^{2} W_{T}^{2}}{\partial z^{2}}=-G_{0} \frac{\partial V^{0}}{\partial n}+\nabla_{\Gamma_{0}} q^{0}+(z-\kappa) \frac{\partial V^{0}}{\partial n} \text { in } \mathbb{R}^{+} \times \Gamma \times[0,1] \\
\frac{\partial W_{T}^{2}}{\partial z}(t, \sigma, z=0)=-\left(\frac{\partial V^{1}}{\partial n}\right)_{T}(t, \sigma) \\
W_{T}^{2}(t, \sigma, z=\kappa)=0
\end{array}\right.
$$

hence

$$
\begin{aligned}
W_{T}^{2}(t, \sigma, z)= & \frac{1}{6}(z-\kappa)^{3} \frac{\partial V^{0}}{\partial n}+\frac{1}{2}(z-\kappa)^{2}\left(-G_{0} \frac{\partial V^{0}}{\partial n}+\nabla_{\Gamma_{0}} q^{0}\right) \\
& -(z-\kappa)\left(\left(\frac{\partial V^{1}}{\partial n}\right)_{T}+\frac{\kappa^{2}}{2} \frac{\partial V^{0}}{\partial n}+\kappa G_{0} \frac{\partial V^{0}}{\partial n}-\kappa \nabla_{\Gamma_{0}} q^{0}\right)
\end{aligned}
$$

Step 6 : we remark that $\int_{\Gamma}\left(W^{2} \cdot n\right) d \sigma=0$, since :

$$
\int_{\Gamma} W_{N}^{2}=\int_{z=0}^{\kappa}\left(\int_{\Gamma} \operatorname{div}_{\Gamma} W_{T}^{1}(\sigma, z) d \sigma\right) d z=0
$$

Thus we can define the extension $V^{2}$ of $W^{2}$ satisfying :

$$
\left\{\begin{array}{l}
V^{2}=W^{2} \text { on } \Gamma \\
V^{2}=0 \text { on } \partial \Omega \\
\operatorname{div} V^{2}=0 \text { in } \mathcal{U}
\end{array}\right.
$$


Step 7 : with (4) at order $\varepsilon^{2}$ we obtain that :

$$
\frac{\partial W_{N}^{3}}{\partial z}+G_{0} W_{N}^{2}+\operatorname{div}_{\Gamma_{0}} W_{T}^{2}+z \operatorname{div}_{\Gamma_{0}}^{\prime} W_{T}^{1}=0
$$

with :

$$
\operatorname{div}_{\Gamma_{0}}^{\prime} Z=-G_{0} \operatorname{div}_{\Gamma} Z+\operatorname{div}_{\Gamma}(G(0) Z-d n(\sigma) Z) .
$$

We define then $W_{N}^{3}$ by :

$$
W_{N}^{3}(\sigma, z)=\int_{z}^{\kappa}\left(G_{0}(\sigma) W_{N}^{2}(\sigma, s)+\operatorname{div}_{\Gamma_{0}} W_{T}^{2}(\sigma)+s \operatorname{div}_{\Gamma_{0}}^{\prime} W_{T}^{1}(\sigma, s)\right) d s .
$$

Step 8 : with (2) at order $\varepsilon$ we have

$$
\begin{aligned}
\frac{\partial W^{1}}{\partial t}-\frac{\partial^{2} W^{3}}{\partial z^{2}}-G_{0} \frac{\partial W^{2}}{\partial z}-z G_{0}^{\prime} \frac{\partial W^{1}}{\partial z}- & \Delta_{\Gamma} W^{1}+W_{N}^{1} \frac{\partial W^{1}}{\partial z}+\frac{\partial q^{2}}{\partial z} n \\
& +\nabla_{\Gamma} q^{1}+z \nabla_{\Gamma}^{\prime} q^{0}+W^{2}=0 .
\end{aligned}
$$

Taking the normal part of this equation we obtain since $W^{1}$ is tangential :

$$
-\frac{\partial^{2} W_{N}^{3}}{\partial z^{2}}-G_{0} \frac{\partial W_{N}^{2}}{\partial z}+\frac{\partial q^{2}}{\partial z}+W_{N}^{2}=0
$$

so we can determine $\frac{\partial q^{2}}{\partial z}$.

Writing (7) at order $\varepsilon^{2}$ we have :

$$
-\frac{\partial W^{3}}{\partial z}+q^{2} n=p^{2} n-\frac{\partial V^{2}}{\partial n} \text { at } z=0 .
$$

Taking the scalar product with $n$, we obtain the value of $q^{2}$ at $z=0$ :

$$
q^{2}(\sigma, z=0)=p^{2}(\sigma)-\left(\frac{\partial V^{2}}{\partial n}\right)_{N}(\sigma)+\left(\frac{\partial W_{N}^{3}}{\partial z}\right)(z=0)
$$

thus $q^{2}$ is completely defined.

Taking now the tangential part of (3.9) we obtain that $W_{T}^{3}$ is completely defined by :

$$
\left\{\begin{array}{l}
\frac{\partial^{2} W_{T}^{3}}{\partial z^{2}}=\frac{\partial W^{1}}{\partial t}-G_{0} \frac{\partial W_{T}^{2}}{\partial z}-\Delta W^{1}+\nabla_{\Gamma} q^{1}+z \nabla_{\Gamma}^{\prime} q^{0}+W_{T}^{2} \\
W_{T}^{3}(t, \sigma, z=\kappa)=0 \\
\frac{\partial W_{T}^{3}}{\partial z}(t, \sigma, z=0)=-\left(\frac{\partial V^{2}}{\partial n}\right)_{T}(t, \sigma)
\end{array}\right.
$$

Step 9 : knowing $W^{3}$ we fix $V^{3}$ satisfying :

$$
\left\{\begin{array}{l}
V^{3}(x)=W^{3}(x, 0) \text { for } x \in \partial \omega \\
V^{3}=0 \text { on } \partial \Omega \\
\operatorname{div} V^{3}=0 \text { in } \mathcal{U}
\end{array}\right.
$$


Remark 3.6 The existence of $V^{3}$ is assured by the fact that $\int_{\Gamma}\left(V^{3} \cdot n\right)=0$ since :

$$
\begin{aligned}
\int_{\Gamma}\left(V^{3} \cdot n\right)= & \int_{z=0}^{\kappa} \int_{\Gamma}\left(G_{0}(\sigma) W_{N}^{2}(\sigma, z)+\operatorname{div}_{\Gamma} W_{T}^{2}(\sigma, z)+z \operatorname{div}_{\Gamma}^{\prime} W_{T}^{1}(\sigma, z)\right) d z d \sigma \\
= & \int_{0}^{\kappa} \int_{\Gamma}\left(G_{0}(\sigma) W_{N}^{2}(\sigma, s)+s \operatorname{div}_{\Gamma}^{\prime} W_{T}^{1}(\sigma, s)\right) d s d \sigma \\
= & \int_{z=0}^{\kappa} \int_{\Gamma} \int_{s=z}^{\kappa} G_{0}(\sigma) \operatorname{div}_{\Gamma} W_{T}^{1}(\sigma, s) d s d z d \sigma-\int_{z=0}^{\kappa} \int_{\Gamma} z G_{0}(\sigma) \operatorname{div}_{\Gamma} W_{T}^{1}(\sigma, z) \\
& +\int_{z=0}^{\kappa} \int_{\Gamma} z \operatorname{div}_{\Gamma}\left(G_{0}(\sigma) W_{T}^{1}(\sigma, z)-d n(\sigma) W_{T}^{1}(\sigma, z)\right) \\
= & \int_{s=0}^{\kappa} \int_{\Gamma} s G_{0}(\sigma) \operatorname{div}_{\Gamma} W_{T}^{1}(\sigma, s) d s d \sigma-\int_{z=0}^{\kappa} \int_{\Gamma} z G_{0}(\sigma) \operatorname{div}_{\Gamma} W_{T}^{1}(\sigma, z) \\
= & 0 .
\end{aligned}
$$

Remark 3.7 we will see in the remainder term estimates that inside $\omega_{\varepsilon}$, we are lead to pusch the asymptotic expansion at order $\varepsilon^{3}$. In $\mathcal{U}$ it is not necessary to be so precise, but to avoid the creation of jumps at the boundary of $\omega_{\varepsilon}$, we have to extend $W^{2}$ and $W^{3}$ with $V^{2}$ and $V^{3}$.

\subsection{Existence and regularity of the terms of the anzatz}

We denote

$$
H=\left\{V \in\left(L^{2}(\mathcal{U})\right)^{3} \text { such that } \operatorname{div} V=0 \text { in } \mathcal{U} \text { and } V \cdot n=0 \text { on } \partial \mathcal{U}\right\}
$$

and

$$
\mathbf{V}=\left\{V \in\left(H_{0}^{1}(\mathcal{U})\right)^{3} \operatorname{such} \text { that } \operatorname{div} V=0 \text { in } \mathcal{U}\right\}
$$

Let $\mathcal{P}$ be the orthogonal projection for the $L^{2}$ scalar product onto $H$.

We denote by $A$ the operator with domain $H \cap H^{2}(\mathcal{U})$ defined by $A=-\mathcal{P} \circ \Delta$, that is if $f \in H$,

$$
A V=f \Longleftrightarrow \exists \pi \in H^{1}(\mathcal{U}) / \mathbb{R}, \quad-\Delta V+\nabla \pi=f .
$$

We recall the results due to Cattabriga (see [9]) :

Proposition 3.1 There exists $C$ such that for all $V \in D(A)$,

$$
\begin{aligned}
& \|V\|_{H^{2}(\mathcal{U})}+\|\pi\|_{H^{1}(\mathcal{U}) / \mathbb{R}} \leq C\|A V\|_{L^{2}(\mathcal{U})}, \\
& \|V\|_{H^{1}(\mathcal{U})}+\|\pi\|_{L^{2}(\mathcal{U}) / \mathbb{R}} \leq C\|A V\|_{H^{-1}(\mathcal{U})} .
\end{aligned}
$$




\subsubsection{Existence of $V^{0}$}

We recall Proposition 1.1. We only give the sketch of the proof of this result. The complete proof can be found in [7].

Proposition 3.2 Let $v_{0} \in H^{8}(\mathcal{U}) \cap \mathbf{V}$. There exists a time $T^{*}>0$ and there exists $V^{0}$ : $\left[0, T^{*}\left[\times \mathcal{U} \longrightarrow \mathbb{R}^{3}\right.\right.$ and $p^{0}:\left[0, T^{*}[\times \mathcal{U} \longrightarrow \mathbb{R}\right.$ such that

$$
\left\{\begin{array}{l}
\frac{\partial V^{0}}{\partial t}-\Delta V^{0}+\left(V^{0} \cdot \nabla\right) V^{0}+\nabla p^{0}=f \quad \text { in }\left[0, T^{*}[\times \mathcal{U}\right. \\
\operatorname{div} V^{0}=0 \quad \text { in }\left[0, T^{*}[\times \mathcal{U}\right. \\
V^{0}=0 \quad \text { on }\left[0, T^{*}[\times \partial \mathcal{U}\right. \\
V^{0}(t=0)=v_{0} \quad \text { in } \mathcal{U} .
\end{array}\right.
$$

For all $T<T^{*}$ and for $0 \leq k \leq 4$,

$$
\frac{\partial^{k} V^{0}}{\partial t^{k}} \in L^{\infty}\left(0, T ; H^{8-2 k}(\mathcal{U})\right) \cap L^{2}\left(0, T ; H^{9-2 k}(\mathcal{U})\right) .
$$

Furthermore for $0 \leq k \leq 3$, the associated presure $p^{0}$ satisfies :

$$
\frac{\partial^{k} p^{0}}{\partial t^{k}} \in L^{\infty}\left(0, T ; H^{7-2 k}(\mathcal{U})\right) \cap L^{2}\left(0, T ; H^{8-2 k}(\mathcal{U})\right) .
$$

Remark 3.8 The regular solution of (3.11) is unique.

Sketch of the proof : we consider a Galerkin approximation of equation (3.11) based on the eigenspaces of the Stokes Operator $A$. We denote by $(3.11)_{a}$ this approximation of (3.11) and by $V_{a}^{0}$ (resp. $p_{a}^{0}$ ) the approximation of $V^{0}$ (resp. $p^{0}$ ) obtained solving $(3.11)_{a}$. Multiplying $(3.11)_{a}$ by $V_{a}^{0}$ and by $A V_{a}^{0}$ we obtain using Gronwall Lemma that there exists $T^{*}$ such that for all $T<T^{*}$, there exists a constant $C$ with :

$$
\left\|V_{a}^{0}\right\|_{L^{\infty}\left(0, T ; H^{1}\right)}+\left\|A V_{a}^{0}\right\|_{L^{2}\left(0, T ; L^{2}\right)} \leq C,
$$

that is using Proposition 3.1

$$
\left\|V_{a}^{0}\right\|_{L^{\infty}\left(0, T ; H^{1}\right)}+\left\|V_{a}^{0}\right\|_{L^{2}\left(0, T ; H^{2}\right)}+\left\|p_{a}^{0}\right\|_{L^{\infty}\left(0, T ; L^{2}\right)}+\left\|p_{a}^{0}\right\|_{L^{2}\left(0, T ; H^{1}\right)} \leq C .
$$

In a second step, derivating $(3.11)_{a}$ with respect to $t$, we obtain the equation satisfied by $\frac{\partial V_{a}^{0}}{\partial t}$. Multiplying this equation by $\frac{\partial V_{a}^{0}}{\partial t}$ and by $A \frac{\partial V_{a}^{0}}{\partial t}$, using Proposition 3.1 we obtain that

$$
\left\|\frac{\partial V_{a}^{0}}{\partial t}\right\|_{L^{\infty}\left(0, T ; H^{1}\right)}+\left\|\frac{\partial V_{a}^{0}}{\partial t}\right\|_{L^{2}\left(0, T ; H^{2}\right)}+\left\|\frac{\partial p_{a}^{0}}{\partial t}\right\|_{L^{\infty}\left(0, T ; L^{2}\right)}+\left\|\frac{\partial p_{a}^{0}}{\partial t}\right\|_{L^{2}\left(0, T ; H^{1}\right)} \leq C .
$$

In a third step we rederivate $(3.11)_{a}$ with respect to $t$ and in the same way we obtain an estimate on $\frac{\partial^{2} V_{a}^{0}}{\partial t^{2}}$ :

$$
\left\|\frac{\partial^{2} V_{a}^{0}}{\partial t^{2}}\right\|_{L^{\infty}\left(0, T ; H^{1}\right)}+\left\|\frac{\partial^{2} V_{a}^{0}}{\partial t^{2}}\right\|_{L^{2}\left(0, T ; H^{2}\right)}+\left\|\frac{\partial^{2} p_{a}^{0}}{\partial t^{2}}\right\|_{L^{\infty}\left(0, T ; L^{2}\right)}+\left\|\frac{\partial^{2} p_{a}^{0}}{\partial t^{2}}\right\|_{L^{2}\left(0, T ; H^{1}\right)} \leq C .
$$


Using these estimates and the equation satisfied by $\frac{\partial V_{a}^{0}}{\partial t}$ we obtain that $A \frac{\partial V_{a}^{0}}{\partial t}$ is bounded in $L^{\infty}\left(0, T ; H^{1}\right) \cap L^{2}\left(0, T ; H^{2}\right)$ hence using Proposition (3.1), there exists a time $T^{*}$ such that for $T<T^{*}$ there exists $\mathrm{C}$ such that

$$
\left\|\frac{\partial V_{a}^{0}}{\partial t}\right\|_{L^{\infty}\left(0, T ; H^{3}\right)}+\left\|\frac{\partial V_{a}^{0}}{\partial t}\right\|_{L^{2}\left(0, T ; H^{4}\right)}+\left\|\frac{\partial p_{a}^{0}}{\partial t}\right\|_{L^{\infty}\left(0, T ; H^{2}\right)}+\left\|\frac{\partial p_{a}^{0}}{\partial t}\right\|_{L^{2}\left(0, T ; H^{3}\right)} \leq C .
$$

In the same way, using this estimate and the equation satisfied by $V_{a}^{0}$ we obtain that

$$
\left\|V_{a}^{0}\right\|_{L^{\infty}\left(0, T ; H^{5}\right)}+\left\|V_{a}^{0}\right\|_{L^{2}\left(0, T: H^{4}\right)}+\left\|p_{a}^{0}\right\|_{L^{\infty}\left(0, T ; H^{4}\right)}+\left\|p_{a}^{0}\right\|_{L^{2}\left(0, T ; H^{5}\right)} \leq C .
$$

Using the same method of derivation in time, we prove first that $\frac{\partial^{3} V_{a}^{0}}{\partial t^{3}}$ is bounded in $L^{\infty}\left(0, T ; H^{1}(\mathcal{U})\right) \cap$ $L^{2}\left(0, T ; H^{2}(\mathcal{U})\right)$ and after that $\frac{\partial^{4} V_{a}^{0}}{\partial t^{4}}$ is bounded in $L^{2}\left(0, T ; L^{2}(\mathcal{U})\right) \cap L^{2}\left(0, T ; H^{1}(\mathcal{U})\right)$. Using Proposition 3.1 and using the equation satisfied by $V_{a}^{0}$ we obtain that:

$$
V_{a}^{0} \text { is bounded in } L^{\infty}\left(0, T ; H^{8}(\mathcal{U})\right) \cap L^{2}\left(0, T ; H^{9}(\mathcal{U})\right)
$$

and for $i \in\{1, \ldots, 4\}$,

$$
\frac{\partial^{i} V_{a}^{0}}{\partial t^{i}} \text { is bounded in } L^{\infty}\left(0, T ; H^{8-2 i}(\mathcal{U})\right) \cap L^{2}\left(0, T ; H^{9-2 i}(\mathcal{U})\right.
$$

and concerning $p_{a}^{0}$,

$$
p_{a}^{0} \text { is bounded in } L^{\infty}\left(0, T ; H^{7}(\mathcal{U})\right) \cap L^{2}\left(0, T ; H^{8}(\mathcal{U})\right)
$$

and for all $i \in\{1,2,3\}$,

$$
\frac{\partial^{i} p_{a}^{0}}{\partial t^{i}} \text { is bounded in } L^{\infty}\left(0, T ; H^{7-2 i}(\mathcal{U})\right) \cap L^{2}\left(0, T ; H^{8-2 i}(\mathcal{U})\right)
$$

Since the bounds do not depend on the dimension of the approximation Galerkin space, taking the weak limit, we obtain the existence of $V^{0}$ and $p^{0}$ which satisfy the same estimates.

Proposition 3.3 Let $V^{0}$ given by Proposition 3.2. Then $\frac{\partial V^{0}}{\partial n}$ is tangential on $\Gamma$.

Proof : as in $\omega_{\varepsilon}$ we build in a neigbourhood of $\Gamma$ in $\mathcal{U}$ a normal parametrization considering $\Phi: \Gamma \times[0, \delta[\rightarrow \mathcal{U}$ defined by :

$$
\Psi(\sigma, z)=\sigma-z n(\sigma) .
$$

(we recall that $n(\sigma)$ is the normal outing from $\mathcal{U}$.

We denote $\tilde{V}^{0}=V^{0} \circ \Phi$ and $\tilde{V}_{N}^{0}$ (resp. $\tilde{V}_{T}^{0}$ ) the normal part (resp. the tangential part) of $\tilde{V}^{0}$.

In the new coordinates $(\sigma, z)$ we have :

$$
0=\operatorname{div} V^{0}=\frac{\partial \tilde{V}_{N}^{0}}{\partial z}+G_{z} \tilde{V}_{N}^{0}+\operatorname{div}_{\Gamma_{z}} \tilde{V}_{T}^{0}
$$

On $\Gamma, \tilde{V}^{0}=0$ hence, $\operatorname{div}_{\Gamma} \tilde{V}_{T}^{0}=0$ and $\tilde{V}_{N}^{0}=0$ on $\Gamma$. Thus on $\Gamma$,

$$
0=\frac{\partial \tilde{V}_{N}^{0}}{\partial z}=\left(\frac{\partial V^{0}}{\partial n}\right)_{N}
$$


3.2.2 Regularity for $W^{1}$ and $q^{0}$

We define $\left.W^{1}:\right] 0, T^{\star}[\times \Gamma \times[0, \kappa] \longrightarrow \mathbb{R}$ by the formula :

$$
W^{1}(\sigma, z)=(z-\kappa) \frac{\partial V^{0}}{\partial n}(t, \sigma)
$$

where $V^{0}$ is given by Proposition 3.2.

On the other hand we define $q^{0}$ by $q^{0}(\sigma, z)=p^{0}(\sigma)$.

Using the results of regularity concerning $V^{0}$ and $p^{0}$ (see Proposition 3.2), we have the following proposition :

Proposition 3.4 Under the hypothesis of Proposition 3.2, for all $T<T^{\star}$, for $0 \leq k \leq 3$,

$$
\frac{\partial^{k} W^{1}}{\partial t^{k}} \in\left(L^{\infty}\left(0, T ; H^{\frac{13-4 k}{2}}(\Gamma)\right) \cap L^{2}\left(0, T ; H^{\frac{15-4 k}{2}}(\Gamma)\right)\right) \otimes \mathcal{C}^{\infty}([0, \kappa])
$$

and

$$
\frac{\partial^{k} q^{0}}{\partial t^{k}} \in\left(L^{\infty}\left(0, T ; H^{\frac{13-4 k}{2}}(\Gamma)\right) \cap L^{2}\left(0, T ; H^{\frac{15-4 k}{2}}(\Gamma)\right)\right) \otimes \mathcal{C}^{\infty}([0, \kappa])
$$

\subsubsection{Existence of $V^{1}$}

We have the following result :

Proposition 3.5 Under the hypothesis of proposition 3.2, there exists $V^{1}:\left[0, T^{\star}\left[\times \mathcal{U} \longrightarrow \mathbb{R}^{3}\right.\right.$ and $p^{1}:\left[0, T^{\star}[\times \mathcal{U} \longrightarrow \mathbb{R}\right.$ such that :

$$
\left\{\begin{array}{l}
\frac{\partial V^{1}}{\partial t}-\Delta V^{1}+\left(V^{0} \cdot \nabla\right) V^{1}+\left(V^{1} \cdot \nabla\right) V^{0}+\nabla p^{1}=0 \quad \text { in } \mathbb{R}^{+} \times \mathcal{U}, \\
\operatorname{div} V^{1}=0 \quad \text { in } \mathbb{R}^{+} \times \mathcal{U}, \\
V^{1}(t, x)=W^{1}(t, x, 0)=-\kappa \frac{\partial V^{0}}{\partial n} \quad \text { on } \mathbb{R}^{+} \times \partial \omega,
\end{array}\right.
$$

and satisfying for $T<T^{\star}$ and for $0 \leq k \leq 2$ :

$$
\left\{\begin{array}{l}
\frac{\partial^{k} V^{1}}{\partial t^{k}} \in L^{\infty}\left(0, T ; H^{5-2 k}(\mathcal{U})\right) \cap L^{2}\left(0, T ; H^{6-2 k}(\mathcal{U})\right) \\
\frac{\partial^{k} p^{1}}{\partial t^{k}} \in L^{\infty}\left(0, T ; L^{4-2 k}(\mathcal{U})\right) \cap L^{2}\left(0, T ; H^{5-2 k}(\mathcal{U})\right)
\end{array}\right.
$$

Proof. We consider $\Upsilon^{1}$ the extension of $W^{1}$ satisfying :

$$
\left\{\begin{array}{l}
\Upsilon^{1}(t, x)=W^{1}(t, x, 0) \text { for } x \in \Gamma \\
\Upsilon^{1}(t, x)=0 \text { for } x \in \partial \Omega \\
\left.-\Delta \Upsilon^{1}+\nabla \Pi^{1}=0 \text { in }\right] 0, T^{\star}[\times \mathcal{U} \\
\left.\operatorname{div} \Upsilon^{1}=0 \text { in }\right] 0, T^{\star}[\times \mathcal{U}
\end{array}\right.
$$


The regularity of $W^{1}$ and its derivatives in times gives that for all $T<T^{\star}$,

$$
\Upsilon^{1} \in L^{\infty}\left(0, T ; H^{7}(\mathcal{U})\right) \cap L^{2}\left(0, T ; H^{8}(\mathcal{U})\right)
$$

and for all $i \in\{1,2,3\}$,

$$
\frac{\partial^{i} \Upsilon^{1}}{\partial t^{i}} \in L^{\infty}\left(0, T ; H^{7-2 i}(\mathcal{U})\right) \cap L^{2}\left(0, T ; H^{8-2 i}(\mathcal{U})\right) .
$$

We will seek $V^{1}$ on the form $V^{1}=\Upsilon^{1}+Z^{1}$ where $Z^{1}$ satisfies :

$$
\left\{\begin{array}{l}
\frac{\partial Z^{1}}{\partial t}-\Delta Z^{1}+\left(V^{0} \cdot \nabla\right) Z^{1}+\left(Z^{1} \cdot \nabla\right) V^{0}+\nabla p^{1}=Q^{1} \\
\operatorname{div} Z^{1}=0 \\
Z^{1}=0 \text { on } \partial \mathcal{U}
\end{array}\right.
$$

with

$$
Q^{1}=-\left(\frac{\partial \Upsilon^{1}}{\partial t}+\left(V^{0} \cdot \nabla\right) \Upsilon^{1}+\left(\Upsilon^{1} \cdot \nabla\right) V^{0}\right)
$$

We remark that for $0 \leq k \leq 2$,

$$
\frac{\partial^{k} Q^{1}}{\partial t^{k}} \in L^{\infty}\left(0, T ; H^{5-2 k}(\mathcal{U})\right) \cap L^{2}\left(0, T ; H^{6-2 k}(\mathcal{U})\right) .
$$

As in the proof of Proposition 3.2 we consider a Galerkin approximation of (3.15). We multiply this approximation by $A Z^{1}$ and we obtain with Proposition 3.1 that :

$$
Z^{1} \in L^{\infty}\left(0, T ; H^{1}(\mathcal{U})\right) \cap L^{2}\left(0, T ; H^{2}(\mathcal{U})\right)
$$

We derivate in time the approximation of (3.15) and multiplying this new equation by $A \frac{\partial Z^{1}}{\partial t}$ we obtain that

$$
\frac{\partial Z^{1}}{\partial t} \in L^{\infty}\left(0, T ; H^{1}(\mathcal{U})\right) \cap L^{2}\left(0, T ; H^{2}(\mathcal{U})\right)
$$

thus using (3.15),

$$
Z^{1} \in L^{\infty}\left(0, T ; H^{3}(\mathcal{U})\right) \cap L^{2}\left(0, T ; H^{4}(\mathcal{U})\right)
$$

Now rederivating in time Equation 3.15 and using the same process we obtain the desired regularity result on $Z^{1}$ and so on $V^{1}$.

\subsubsection{Regularity of $W^{2}$ and $W^{3}$}

We define $W^{2}:\left[0, T^{\star}[\times \Gamma \times[0, \kappa]\right.$ by :

$$
W_{N}^{2}(t, \sigma, z)=-\frac{1}{2}(z-\kappa)^{2} \operatorname{div} \Gamma_{\Gamma}\left(\frac{\partial V^{0}}{\partial n}\right)
$$

and

$$
\begin{aligned}
W_{T}^{2}(t, \sigma, z)= & \frac{1}{6}(z-\kappa)^{3} \frac{\partial V^{0}}{\partial n}+\frac{1}{2}(z-\kappa)^{2}\left(-G_{0} \frac{\partial V^{0}}{\partial n}+\nabla_{\Gamma_{0}} q^{0}\right) \\
& -(z-\kappa)\left(\left(\frac{\partial V^{1}}{\partial n}\right)_{T}+\frac{\kappa^{2}}{2} \frac{\partial V^{0}}{\partial n}+\kappa G_{0} \frac{\partial V^{0}}{\partial n}-\kappa \nabla_{\Gamma_{0}} q^{0}\right)
\end{aligned}
$$


and with the regularity proved for $q^{0}, W^{1}$ and $V^{1}$ we obtain that :

$$
\left\{\begin{array}{l}
W^{2} \in\left(L^{\infty}\left(0, T ; H^{\frac{7}{2}}(\Gamma)\right) \cap L^{2}\left(0, T ; H^{\frac{9}{2}}(\Gamma)\right)\right) \otimes \mathcal{C}^{\infty}([0,1]) \\
\frac{\partial W^{2}}{\partial t} \in\left(L^{\infty}\left(0, T ; H^{\frac{3}{2}}(\Gamma)\right) \cap L^{2}\left(0, T ; H^{\frac{5}{2}}(\Gamma)\right)\right) \otimes \mathcal{C}^{\infty}([0,1])
\end{array}\right.
$$

Now $W^{3}$ is defined by (3.8) and (3.10), and it is a polynomial map in the $z$ variable with coefficients depending on $t$ and $\sigma$, obtained with the previous profiles.

Therefore we obtain that

$$
\left\{\begin{array}{l}
W^{3} \in\left(L^{\infty}\left(0, T ; H^{\frac{5}{2}}(\Gamma)\right) \cap L^{2}\left(0, T ; H^{\frac{7}{2}}(\Gamma)\right)\right) \otimes \mathcal{C}^{\infty}([0,1]) \\
\frac{\partial W^{3}}{\partial t} \in\left(L^{\infty}\left(0, T ; H^{\frac{1}{2}}(\Gamma)\right) \cap L^{2}\left(0, T ; H^{\frac{3}{2}}(\Gamma)\right)\right) \otimes \mathcal{C}^{\infty}([0,1])
\end{array}\right.
$$

\subsubsection{Regularity of $V^{2}$ and $V^{3}$}

With the regularity obtained for $W^{2}$ and $W^{3}$, their extensions $V^{2}$ and $V^{3}$ satisfy :

$$
\left\{\begin{array}{l}
V^{2} \in L^{\infty}\left(0, T ; H^{4}(\mathcal{U})\right) \cap L^{2}\left(0, T ; H^{5}(\mathcal{U})\right) \\
\frac{\partial V^{2}}{\partial t} \in L^{\infty}\left(0, T ; H^{2}(\mathcal{U})\right) \cap L^{2}\left(0, T ; H^{3}(\mathcal{U})\right) \\
V^{3} \in L^{\infty}\left(0, T ; H^{3}(\mathcal{U})\right) \cap L^{2}\left(0, T ; H^{4}(\mathcal{U})\right) \\
\frac{\partial V^{3}}{\partial t} \in L^{\infty}\left(0, T ; H^{1}(\mathcal{U})\right) \cap L^{2}\left(0, T ; H^{2}(\mathcal{U})\right)
\end{array}\right.
$$

\section{Estimate of the remainder term for Theorem 1.1}

We define the different terms of the ansatz as in the previous section and we introduce the approximations $W^{\varepsilon}$ of the velocity $w^{\varepsilon}$ in $\omega_{\varepsilon}$ and his approximation $V^{\varepsilon}$ in $\mathcal{U}$ defined as follows :

$$
W^{\varepsilon}(t, x)=\varepsilon W^{1}\left(t, P(x), \frac{\varphi(x)}{\varepsilon}\right)+\varepsilon^{2} W^{2}\left(t, P(x), \frac{\varphi(x)}{\varepsilon}\right)+\varepsilon^{3} W^{3}\left(t, P(x), \frac{\varphi(x)}{\varepsilon}\right)
$$

and

$$
V^{\varepsilon}=V^{0}(t, x)+\varepsilon V^{1}(t, x)+\varepsilon^{2} V^{2}(t, x)+\varepsilon^{3} V^{3}(t, x)
$$

We set :

$$
\begin{aligned}
& w^{\varepsilon}(t, x)=W^{\varepsilon}(t, x)+\varepsilon^{2} w_{\varepsilon}^{r}(t, x) \\
& v^{\varepsilon}(t, x)=V^{\varepsilon}(t, x)+\varepsilon^{2} v_{\varepsilon}^{r}(t, x) \\
& q^{\varepsilon}(t, x)=q^{0}\left(t, P(x), \frac{\varphi(x)}{\varepsilon}\right)+\varepsilon q^{1}\left(t, P(x), \frac{\varphi(x)}{\varepsilon}\right)+\varepsilon^{2} q_{\varepsilon}^{r}(t, x) \\
& p^{\varepsilon}(t, x)=p^{0}(t, x)+\varepsilon p^{1}(t, x)+\varepsilon^{2} p_{\varepsilon}^{r}(t, x) .
\end{aligned}
$$

We will write the equations satisfied by the remainder terms in order to estimate them. 


$$
\left\{\begin{array}{lr}
\frac{\partial w_{\varepsilon}^{r}}{\partial t}-\Delta w_{\varepsilon}^{r}+\left(w_{\varepsilon}^{r} \cdot \nabla\right) W^{\varepsilon}+\varepsilon^{2}\left(w_{\varepsilon}^{r} \cdot \nabla\right) w_{\varepsilon}^{r}+\left(W^{\varepsilon} \cdot \nabla\right) w_{\varepsilon}^{r} & \text { in } \mathbb{R}^{+} \times \omega_{\varepsilon} \\
+\nabla q_{\varepsilon}^{r}+\frac{1}{\varepsilon} w_{\varepsilon}^{r}=R_{\text {porous }}^{\varepsilon} & \\
\operatorname{div} w_{\varepsilon}^{r}=g_{\varepsilon} & \text { in } \mathbb{R}^{+} \times \omega_{\varepsilon} \\
\frac{\partial v_{\varepsilon}^{r}}{\partial t}-\Delta v_{\varepsilon}^{r}+\left(V^{\varepsilon} \cdot \nabla\right) v_{\varepsilon}^{r}+\left(v_{\varepsilon}^{r} \cdot \nabla\right) V^{\varepsilon}+\varepsilon^{2}\left(v_{\varepsilon}^{r} \cdot \nabla\right) v_{\varepsilon}^{r} & \text { in } \mathbb{R}^{+} \times \mathcal{U} \\
+\nabla p_{\varepsilon}^{r}=R_{f l u}^{\varepsilon} & \\
\operatorname{div} v_{\varepsilon}^{r}=0 & \text { in } \mathbb{R}^{+} \times \mathcal{U} \\
v_{\varepsilon}^{r}=w_{\varepsilon}^{r} & \text { in } \mathbb{R}^{+} \times \Gamma \\
-\frac{\partial v_{\varepsilon}^{r}}{\partial n}+p_{\varepsilon}^{r} n+\frac{\partial w_{\varepsilon}^{r}}{\partial n}-q_{\varepsilon}^{r} n=R_{\text {bound }}^{\varepsilon} & \text { in } \mathbb{R}^{+} \times \Gamma
\end{array}\right.
$$

where :

$$
\begin{aligned}
R_{\text {porous }}^{\varepsilon}= & \frac{1}{\varepsilon^{2}}\left[\left(G_{\varphi(x)}-G_{0}-\varphi(x) G_{0}^{\prime}\right) \frac{\partial W^{1}}{\partial z}-\left(\nabla_{\Gamma_{\varphi(x)}}-\nabla_{\Gamma}-\varphi(x) \nabla_{\Gamma}^{\prime}\right) q^{0}\right] \\
& +\frac{1}{\varepsilon}\left[\left(G_{\varphi(x)}-G_{0}\right) \frac{\partial W^{2}}{\partial z}+\left(\Delta_{\Gamma_{\varphi(x)}}-\Delta_{\Gamma}\right) W^{1}-\left(\nabla_{\Gamma_{\varphi(x)}}-\nabla_{\Gamma}\right) q^{1}\right] \\
& -\frac{\partial W^{2}}{\partial t}-\varepsilon \frac{\partial W^{3}}{\partial t}+G_{\varphi(x)} \frac{\partial W^{3}}{\partial z}+\varepsilon \nabla_{\Gamma_{\varphi(x)}} W^{3}+\Delta_{\Gamma_{\varphi(x)}} W^{2}-W^{3} \\
& -\frac{1}{\varepsilon^{2}}\left(W^{\varepsilon} \cdot \nabla\right) W^{\varepsilon}
\end{aligned}
$$




$$
\begin{aligned}
g_{\varepsilon}= & -\frac{1}{\varepsilon^{2}} \operatorname{div} W^{\varepsilon} \\
= & -\varepsilon G_{\varphi(x)} W_{N}^{3}-\left(G_{\varphi(x)}-G_{0}\right) W_{N}^{2}-\left(\operatorname{div}_{\Gamma_{\varphi(x)}}-\operatorname{div}_{\Gamma_{0}}\right) W_{T}^{2} \\
& -\frac{1}{\varepsilon}\left[\operatorname{div}_{\Gamma_{\varphi(x)}}-\operatorname{div}_{\Gamma_{0}}-\varphi(x) \operatorname{div}_{\Gamma_{0}}^{\prime}\right] W_{T}^{1} \\
R_{f l u}^{\varepsilon}= & -\left(\frac{\partial V^{2}}{\partial t}-\Delta V^{2}+\left(V^{1} \cdot \nabla\right) V^{1}+\left(V^{0} \cdot \nabla\right) V^{2}+\left(V^{2} \cdot \nabla\right) V^{0}\right) \\
& -\varepsilon\left(\frac{\partial V^{3}}{\partial t}-\Delta V^{3}+\left(V^{0} \cdot \nabla\right) V^{3}+\left(V^{1} \cdot \nabla\right) V^{2}+\left(V^{2} \cdot \nabla\right) V^{1}+\left(V^{3} \cdot \nabla\right) V^{0}\right) \\
& -\varepsilon^{2}\left(\left(V^{1} \cdot \nabla\right) V^{3}+\left(V^{2} \cdot \nabla\right) V^{2}+\left(V^{3} \cdot \nabla\right) V^{1}\right)-\varepsilon^{3}\left(\left(V^{2} \cdot \nabla\right) V^{3}+\left(V^{3} \cdot \nabla\right) V^{2}\right) \\
& -\varepsilon^{4}\left(V^{3} \cdot \nabla\right) V^{3} \\
R_{\text {bound }}^{\varepsilon}= & \varepsilon \frac{\partial V^{3}}{\partial n}
\end{aligned}
$$

Lemma 4.1 We have the following estimates : for $T<T^{\star}$ there exists $C$ such that

$$
\left\{\begin{array}{l}
\left\|W^{\varepsilon}\right\|_{L^{\infty}\left(0, T ; W^{1, \infty}\left(\omega_{\varepsilon}\right)\right)} \leq C \\
\left\|V^{\varepsilon}\right\|_{L^{\infty}\left(0, T ; W^{1, \infty}(\mathcal{U})\right)} \leq C \\
\left\|R_{\text {porous }}^{\varepsilon}\right\|_{L^{2}\left(0, T ; L^{2}\left(\omega_{\varepsilon}\right)\right)} \leq C \\
\left\|g_{\varepsilon}\right\|_{L^{\infty}\left(0, T ; L^{2}\left(\omega_{\varepsilon}\right)\right)} \leq C \varepsilon \\
\left\|R_{\text {flu }}^{\varepsilon}\right\|_{L^{2}\left(0, T ; L^{2}(\mathcal{U})\right)} \leq C \\
\left\|R_{\text {bound }}^{\varepsilon}\right\|_{L^{2}\left(0, T ; L^{2}(\Gamma)\right)} \leq C
\end{array}\right.
$$

Proof : these estimates are direct consequences of the regularity results proved for the different terms of the asymptotic expansion.

In order to estimate the term $\left(w_{\varepsilon}^{r} \cdot \nabla\right) w_{\varepsilon}^{r}$ we need a divergence free condition. Since $\left\|g_{\varepsilon}\right\|_{L^{\infty}\left(0, T ; L^{2}(\omega)\right)} \leq$ $C \varepsilon$, using Theorem 2.1 we have the following lemma :

Lemma 4.2 There exists a constant $C$ such that for all $\varepsilon>0$ there exists $\psi_{\varepsilon} \in H_{0}^{1}\left(\omega_{\varepsilon}\right)$ satisfying :

$$
\left\{\begin{array}{l}
\operatorname{div} \psi_{\varepsilon}=g_{\varepsilon} \text { in } \omega_{\varepsilon} \\
\left\|\psi_{\varepsilon}\right\|_{L^{2}\left(\omega_{\varepsilon}\right)} \leq C \varepsilon \\
\left\|\nabla \psi_{\varepsilon}\right\|_{L^{2}\left(\omega_{\varepsilon}\right)} \leq C
\end{array}\right.
$$


We multiply (1) in (4.17) by $w_{\varepsilon}^{r}-\psi_{\varepsilon}$ and we integrate on $\omega_{\varepsilon}$. We obtain :

$$
\frac{1}{2} \frac{d}{d t}\left\|w_{\varepsilon}^{r}\right\|^{2}+\left\|\nabla w_{\varepsilon}^{r}\right\|^{2}+\frac{1}{\varepsilon}\left\|w_{\varepsilon}^{r}\right\|^{2}=I_{1}+\ldots+I_{11}
$$

with

$$
\begin{aligned}
& I_{1}=-\int_{\Gamma} w_{\varepsilon}^{r}\left(\frac{\partial w_{\varepsilon}^{r}}{\partial n}-q_{\varepsilon}^{r} n\right) \\
& I_{2}=-\int_{\omega_{\varepsilon}}\left(\left(w_{\varepsilon}^{r} \cdot \nabla\right) W^{\varepsilon}+\left(W^{\varepsilon} \cdot \nabla\right) w_{\varepsilon}^{r}\right)\left(w_{\varepsilon}^{r}-\psi_{\varepsilon}\right), \\
& I_{3}=-\varepsilon^{2} \int_{\omega_{\varepsilon}}\left(\left(w_{\varepsilon}^{r}-\psi_{\varepsilon}\right) \cdot \nabla\right)\left(w_{\varepsilon}^{r}-\psi_{\varepsilon}\right)\left(w_{\varepsilon}^{r}-\psi_{\varepsilon}\right) \\
& I_{4}=-\varepsilon^{2} \int_{\omega_{\varepsilon}}\left(\left(w_{\varepsilon}^{r} \cdot \nabla\right) \psi_{\varepsilon} \cdot w_{\varepsilon}^{r}+\left(w_{\varepsilon}^{r} \cdot \nabla\right) w_{\varepsilon}^{r} \cdot \psi_{\varepsilon}+\left(\psi_{\varepsilon} \cdot \nabla\right) w_{\varepsilon}^{r} \cdot w_{\varepsilon}^{r}\right) \\
& I_{5}=\varepsilon^{2} \int_{\omega_{\varepsilon}}\left(\left(w_{\varepsilon}^{r} \cdot \nabla\right) \psi_{\varepsilon} \cdot \psi_{\varepsilon}+\left(\psi_{\varepsilon} \cdot \nabla\right) w_{\varepsilon}^{r} \cdot \psi_{\varepsilon}+\left(\psi_{\varepsilon} \cdot \nabla\right) \psi_{\varepsilon} \cdot w_{\varepsilon}^{r}\right), \\
& I_{6}=-\varepsilon^{2} \int_{\omega_{\varepsilon}}\left(\psi_{\varepsilon} \cdot \nabla\right) \psi_{\varepsilon} \cdot \psi_{\varepsilon} \\
& I_{7}=\frac{1}{\varepsilon} \int_{\omega_{\varepsilon}} w_{\varepsilon}^{r} \psi_{\varepsilon}, \quad I_{8}=\int_{\omega_{\varepsilon}} R_{\text {porous }}^{\varepsilon} w_{\varepsilon}^{r} \\
& I_{9}=-\int_{\omega_{\varepsilon}} \frac{\partial w_{\varepsilon}^{r}}{\partial t} \psi_{\varepsilon}, \quad I_{10}=\int_{\omega_{\varepsilon}} \nabla w_{\varepsilon}^{r} \nabla \psi_{\varepsilon} \\
& I_{11}=-\int_{\omega_{\varepsilon}} R_{\text {porous }}^{\varepsilon} \psi^{\varepsilon}
\end{aligned}
$$

We multiply (3) in (4.17) by $v_{\varepsilon}^{r}$ and we obtain that :

$$
\frac{1}{2} \frac{d}{d t}\left\|v_{\varepsilon}^{r}\right\|^{2}+\left\|\nabla v_{\varepsilon}^{r}\right\|^{2}=J_{1}+\ldots+J_{4}
$$


where:

$$
\begin{aligned}
& J_{1}=\int_{\Gamma}\left(\frac{\partial v_{\varepsilon}^{r}}{\partial n} v_{\varepsilon}^{r}-p^{\varepsilon} v_{\varepsilon}^{r} \cdot n\right) \\
& J_{2}=-\int_{\mathcal{U}}\left(\left(V^{\varepsilon} \cdot \nabla\right) v_{\varepsilon}^{r} \cdot v_{\varepsilon}^{r}+\left(v_{\varepsilon}^{r} \cdot \nabla\right) V^{\varepsilon} \cdot v_{\varepsilon}^{r}\right) \\
& J_{3}=-\varepsilon^{2} \int_{\mathcal{U}}\left(v_{\varepsilon}^{r} \cdot \nabla\right) v_{\varepsilon}^{r} \cdot v_{\varepsilon}^{r}, \\
& J_{4}=\int_{\mathcal{U}} R_{f l u}^{\varepsilon} v_{\varepsilon}^{r},
\end{aligned}
$$

We add (4.19) and (4.20). We estimate the right hand side terms in the following way:

$$
\begin{aligned}
& \left|I_{1}+J_{1}\right|=\left|\int_{\Gamma} R_{\text {bound }}^{\varepsilon} w_{\varepsilon}^{r}\right| \\
& \leq\left\|R_{\text {bound }}^{\varepsilon}\right\|_{L^{2}(\Gamma)}\left\|w_{\varepsilon}^{r}\right\|_{L^{2}(\Gamma)} \\
& \leq C\left\|w_{\varepsilon}^{r}\right\|_{L^{2}\left(\omega_{\varepsilon}\right)}^{\frac{1}{2}}\left\|\nabla w_{\varepsilon}^{r}\right\|_{L^{2}\left(\omega_{\varepsilon}\right)}^{\frac{1}{2}}+\frac{C}{\sqrt{\varepsilon}}\left\|w_{\varepsilon}^{r}\right\|_{L^{2}\left(\omega_{\varepsilon}\right)} \\
& \leq \frac{1}{10 \varepsilon}\left\|w_{\varepsilon}^{r}\right\|_{L^{2}\left(\omega_{\varepsilon}\right)}^{2}+K+\frac{1}{10}\left\|\nabla w_{\varepsilon}^{r}\right\|_{L^{2}\left(\omega_{\varepsilon}\right)}^{2}+\left\|w_{\varepsilon}^{r}\right\|_{L^{2}\left(\omega_{\varepsilon}\right)}^{2} \\
& \left|I_{2}\right| \leq\left\|w_{\varepsilon}^{r}\right\|_{L^{2}\left(\omega_{\varepsilon}\right)}\left\|\nabla W^{\varepsilon}\right\|_{L^{\infty}\left(\omega_{\varepsilon}\right)}\left(\left\|w_{\varepsilon}^{r}\right\|_{L^{2}\left(\omega_{\varepsilon}\right)}+\left\|\psi_{\varepsilon}\right\|_{L^{2}\left(\omega_{\varepsilon}\right)}\right) \\
& +\left\|W^{\varepsilon}\right\|_{L^{\infty}\left(\omega_{\varepsilon}\right)}\left\|\nabla w_{\varepsilon}^{r}\right\|_{L^{2}\left(\omega_{\varepsilon}\right)}\left(\left\|w_{\varepsilon}^{r}\right\|_{L^{2}\left(\omega_{\varepsilon}\right)}+\left\|\psi_{\varepsilon}\right\|_{L^{2}\left(\omega_{\varepsilon}\right)}\right) \\
& \leq \frac{1}{10}\left\|\nabla w_{\varepsilon}^{r}\right\|_{L^{2}\left(\omega_{\varepsilon}\right)}^{2}+K\left\|w_{\varepsilon}^{r}\right\|_{L^{2}\left(\omega_{\varepsilon}\right)}^{2}+K \\
& I_{3}=-\varepsilon^{2} \sum_{i, j} \int_{\omega_{\varepsilon}}\left(w_{\varepsilon}^{r, i}-\psi_{\varepsilon}^{i}\right) \frac{\partial}{\partial x_{i}}\left(w^{r, j}-\psi_{\varepsilon}^{j}\right)\left(w_{\varepsilon}^{r, j}-\psi_{\varepsilon}^{j}\right) \\
& =-\varepsilon^{2} \sum_{i j} \int_{\omega_{\varepsilon}}\left(w_{\varepsilon}^{r, i}-\psi_{\varepsilon}^{i}\right) \frac{1}{2} \frac{\partial}{\partial x_{i}}\left(\left(w_{\varepsilon}^{r, j}-\psi_{\varepsilon}^{j}\right)^{2}\right) \\
& =-\frac{1}{2} \varepsilon^{2} \sum_{i j} \int_{\Gamma}\left(w_{\varepsilon}^{r, i}-\psi_{\varepsilon}^{i}\right) n_{i}\left(w_{\varepsilon}^{r, j}-\psi_{\varepsilon}^{j}\right)^{2} \\
& +\frac{1}{2} \varepsilon^{2} \int_{\omega_{\varepsilon}}\left(w_{\varepsilon}^{r, j}-\psi_{\varepsilon}^{j}\right)^{2} \frac{\partial}{\partial x_{i}}\left(w_{\varepsilon}^{r, i}-\psi_{\varepsilon}^{i}\right)
\end{aligned}
$$


Using that $\psi_{\varepsilon}=0$ on $\Gamma$ and that $\operatorname{div}\left(w_{\varepsilon}^{r}-\psi_{\varepsilon}\right)=0$, we obtain finally that :

$$
\begin{aligned}
& I_{3}=-\frac{1}{2} \varepsilon^{2} \int_{\Gamma} w_{\varepsilon}^{r} \cdot n\left|w_{\varepsilon}^{r}\right|^{2} d \sigma \\
& \left|I_{4}\right| \leq \varepsilon^{2}\left\|w_{\varepsilon}^{r}\right\|_{L^{6}\left(\omega_{\varepsilon}\right)}\left\|\nabla \psi_{\varepsilon}\right\|_{L^{2}\left(\omega_{\varepsilon}\right)}\left\|w_{\varepsilon}^{r}\right\|_{L^{3}\left(\omega_{\varepsilon}\right)}+2 \varepsilon^{2}\left\|w_{\varepsilon}^{r}\right\|_{L^{3}\left(\omega_{\varepsilon}\right)}\left\|\nabla w_{\varepsilon}^{r}\right\|_{L^{2}\left(\omega_{\varepsilon}\right)}\left\|\psi_{\varepsilon}\right\|_{L^{6}\left(\omega_{\varepsilon}\right)} \\
& \leq C \varepsilon^{2} \varepsilon^{-\frac{1}{3}}\left\|w_{\varepsilon}^{r}\right\|_{H^{1}\left(\omega_{\varepsilon}\right)}\left\|w_{\varepsilon}^{r}\right\|_{L^{2}\left(\omega_{\varepsilon}\right)}^{\frac{1}{2}} \varepsilon^{-\frac{1}{6}}\left\|w_{\varepsilon}^{r}\right\|_{H^{1}\left(\omega_{\varepsilon}\right)}^{\frac{1}{2}} \\
& +C \varepsilon^{2}\left\|\nabla w_{\varepsilon}^{r}\right\|_{L^{2}\left(\omega_{\varepsilon}\right)} \varepsilon^{-\frac{1}{3}}\left\|\psi_{\varepsilon}\right\|_{H^{1}\left(\omega_{\varepsilon}\right)}\left\|w_{\varepsilon}^{r}\right\|_{L^{2}\left(\omega_{\varepsilon}\right)}^{\frac{1}{2}} \varepsilon^{-\frac{1}{6}}\left\|w_{\varepsilon}^{r}\right\|_{H^{1}\left(\omega_{\varepsilon}\right)} \\
& \leq C \varepsilon^{\frac{3}{2}}\left\|\nabla w_{\varepsilon}^{r}\right\|_{L^{2}\left(\omega_{\varepsilon}\right)}^{\frac{3}{2}}\left\|w_{\varepsilon}^{r}\right\|_{L^{2}\left(\omega_{\varepsilon}\right)}^{\frac{1}{2}}+C \varepsilon^{\frac{3}{2}}\left\|w_{\varepsilon}^{r}\right\|_{L^{2}\left(\omega_{\varepsilon}\right)}^{\frac{3}{2}} \\
& \leq \frac{1}{10}\left\|\nabla w_{\varepsilon}^{r}\right\|_{L^{2}\left(\omega_{\varepsilon}\right)}+K\left\|w_{\varepsilon}^{r}\right\|_{L^{2}\left(\omega_{\varepsilon}\right)}+K \\
& \left|I_{5}\right| \leq \varepsilon^{2}\left\|\nabla \psi_{\varepsilon}\right\|_{L^{2}\left(\omega_{\varepsilon}\right)}\left\|w_{\varepsilon}^{r}\right\|_{L^{6}\left(\omega_{\varepsilon}\right)}\left\|\psi_{\varepsilon}\right\|_{L^{3}\left(\omega_{\varepsilon}\right)}+\varepsilon^{2}\left\|w_{\varepsilon}^{r}\right\|_{L^{6}\left(\omega_{\varepsilon}\right)}\left\|\psi_{\varepsilon}\right\|_{L^{3}\left(\omega_{\varepsilon}\right)}\left\|\nabla \psi_{\varepsilon}\right\|_{L^{2}\left(\omega_{\varepsilon}\right)}
\end{aligned}
$$

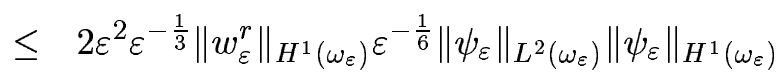

$$
\begin{aligned}
& \leq C \varepsilon^{2}\left(\left\|\nabla w_{\varepsilon}^{r}\right\|_{L^{2}\left(\omega_{\varepsilon}\right)}+\left\|w_{\varepsilon}^{r}\right\|_{L^{2}\left(\omega_{\varepsilon}\right)}\right) \\
& \leq \frac{1}{10}\left\|\nabla w_{\varepsilon}^{r}\right\|_{L^{2}\left(\omega_{\varepsilon}\right)}^{2}+\left\|w_{\varepsilon}^{r}\right\|_{L^{2}\left(\omega_{\varepsilon}\right)}^{2} \\
& \left|I_{6}\right| \leq \varepsilon^{2}\left\|\nabla \psi_{\varepsilon}\right\|_{L^{2}\left(\omega_{\varepsilon}\right)}\left\|\psi_{\varepsilon}\right\|_{L^{3}\left(\omega_{\varepsilon}\right)}\left\|\psi_{\varepsilon}\right\|_{L^{6}\left(\omega_{\varepsilon}\right)} \\
& \leq \varepsilon^{2}\left\|\psi_{\varepsilon}\right\|_{H^{1}\left(\omega_{\varepsilon}\right)}^{3} \leq K \\
& \left|I_{7}\right| \leq \frac{1}{\varepsilon}\left\|w_{\varepsilon}^{r}\right\|_{L^{2}\left(\omega_{\varepsilon}\right)}\left\|\psi_{\varepsilon}\right\|_{L^{2}\left(\omega_{\varepsilon}\right)} \\
& \leq \frac{1}{10 \varepsilon}\left\|w_{\varepsilon}^{r}\right\|_{L^{2}\left(\omega_{\varepsilon}\right)}^{2}+\frac{C}{\varepsilon}\left\|\psi_{\varepsilon}\right\|_{L^{2}\left(\omega_{\varepsilon}\right)}^{2} \\
& \leq \frac{1}{10 \varepsilon}\left\|w_{\varepsilon}^{r}\right\|_{L^{2}\left(\omega_{\varepsilon}\right)}^{2}+K \\
& \left|I_{8}\right| \leq \varepsilon\left\|R_{\text {porous }}^{\varepsilon}\right\|_{L^{2}\left(\omega_{\varepsilon}\right)}^{2}+\frac{1}{10 \varepsilon}\left\|w_{\varepsilon}^{r}\right\|_{L^{2}\left(\omega_{\varepsilon}\right)}^{2} \\
& \leq \frac{1}{10 \varepsilon}\left\|w_{\varepsilon}^{r}\right\|_{L^{2}\left(\omega_{\varepsilon}\right)}^{2}+K
\end{aligned}
$$


We integrate $I_{9}$ in time from 0 to $T$ and we obtain that

$$
\begin{aligned}
\int_{0}^{T} I_{9}= & \int_{\omega_{\varepsilon}} w_{\varepsilon}^{r}(T) \psi_{\varepsilon}(T)-\int_{\omega} w_{\varepsilon}^{r}(0) \psi_{\varepsilon}(0)+\int_{0}^{T} \int_{\omega_{\varepsilon}} w_{\varepsilon}^{r} \frac{\partial \psi_{\varepsilon}}{\partial t} \\
\leq & \frac{1}{9}\left\|w_{\varepsilon}^{r}(T)\right\|_{L^{2}\left(\omega_{\varepsilon}\right)}+W\left\|\psi_{\varepsilon}(T)\right\|_{L^{2}\left(\omega_{\varepsilon}\right)}^{2}+K+\int_{0}^{T}\left\|w_{\varepsilon}^{r}\right\|_{L^{2}\left(\omega_{\varepsilon}\right)}^{2} \\
& +\int_{0}^{T}\left\|\frac{\partial \psi_{\varepsilon}}{\partial t}\right\|_{L^{2}\left(\omega_{\varepsilon}\right)}^{2} \\
\left|I_{10}\right| \leq & \frac{1}{10}\left\|\nabla w_{\varepsilon}^{r}\right\|_{L^{2}\left(\omega_{\varepsilon}\right)}^{2}+C\left\|\psi_{\varepsilon}\right\|_{H^{1}\left(\omega_{\varepsilon}\right)}^{2} \\
\leq & \frac{1}{10}\left\|\nabla w_{\varepsilon}^{r}\right\|_{L^{2}\left(\omega_{\varepsilon}\right)}^{2}+K \\
\left|I_{11}\right| \leq & \left\|R_{\text {porous }}^{\varepsilon}\right\|_{L^{2}\left(\omega_{\varepsilon}\right)}\left\|\psi_{\varepsilon}\right\|_{L^{2}\left(\omega_{\varepsilon}\right)} \leq K
\end{aligned}
$$

We estimate the right hand side terms of (4.20) in the following way :

$$
\begin{aligned}
\left|J_{2}\right| & \leq\left\|V^{\varepsilon}\right\|_{L^{6}(\mathcal{U})}\left\|\nabla v_{\varepsilon}^{r}\right\|_{L^{2}(\mathcal{U})}\left\|v_{\varepsilon}^{r}\right\|_{L^{3}(\mathcal{U})}+\left\|\nabla V^{\varepsilon}\right\|_{L^{2}(\mathcal{U})}\left\|v_{\varepsilon}^{r}\right\|_{L^{4}(\mathcal{U})}^{2} \\
& \leq\left\|V^{\varepsilon}\right\|_{H^{1}(\mathcal{U})}\left\|v_{\varepsilon}^{r}\right\|_{H^{1}(\mathcal{U})}^{\frac{3}{2}}\left\|v_{\varepsilon}^{r}\right\|_{L^{2}(\mathcal{U})}^{\frac{1}{2}} \\
& \leq\left\|V^{\varepsilon}\right\|_{H^{1}(\mathcal{U})}\left(\left\|v_{\varepsilon}^{r}\right\|_{L^{2}(\mathcal{U})}^{2}+\left\|\nabla v_{\varepsilon}^{r}\right\|_{L^{2}(\mathcal{U})}^{\frac{3}{2}}\left\|v_{\varepsilon}^{r}\right\|_{L^{2}(\mathcal{U})}^{\frac{1}{2}}\right) \\
& \leq \frac{1}{10}\left\|\nabla v_{\varepsilon}^{r}\right\|_{L^{2}(\mathcal{U})}^{2}+C\left\|v_{\varepsilon}^{r}\right\|_{L^{2}(\mathcal{U})}^{2}
\end{aligned}
$$

For $J_{3}$ we perform the same calculation as for $I_{3}$ and since $v_{\varepsilon}^{r}=w_{\varepsilon}^{r}$ on $\Gamma$, we obtain that :

$$
\begin{aligned}
J_{3} & =\varepsilon^{2} \frac{1}{2} \int_{\Gamma} v_{\varepsilon}^{r} \cdot n\left|v_{\varepsilon}^{r}\right|^{2} d \sigma=-I_{3}, \\
\left|J_{4}\right| & \leq\left\|R_{f l u}^{\varepsilon}\right\|_{L^{2}(\mathcal{U})}^{2}+\left\|v_{\varepsilon}^{r}\right\|_{L^{2}(\mathcal{U})}^{2} \\
& \leq\left\|v_{\varepsilon}^{r}\right\|_{L^{2}(\mathcal{U})}^{2}+K
\end{aligned}
$$

Hence adding all the previous inequality we obtain that there exists a function $K \in L^{1}(0, T ; \mathbb{R})$ for all $T<T^{*}$, there exists $\alpha_{1}>0$ and $\alpha_{2}>0$ such that :

$$
\frac{d}{d t}\left(\left\|w_{\varepsilon}^{r}\right\|_{L^{2}\left(\omega_{\varepsilon}\right)}^{2}+\left\|v_{\varepsilon}^{r}\right\|_{L^{2}(\mathcal{U})}^{2}\right)+\alpha_{1}\left(\left\|\nabla w_{\varepsilon}^{r}\right\|_{L^{2}\left(\omega_{\varepsilon}\right)}^{2}+\left\|\nabla v_{\varepsilon}^{r}\right\|_{L^{2}(\mathcal{U})}^{2}\right)+\frac{\alpha_{2}}{\varepsilon}\left\|w_{\varepsilon}^{r}\right\|_{L^{2}\left(\omega_{\varepsilon}\right)}^{2} \leq K(t)\left(1+\left\|v_{\varepsilon}^{r}\right\|_{L^{2}(\mathcal{U})}^{2}\right)
$$

We conclude the proof with a classical Gronwall Lemma. 


\section{Proof of Theorem 1.2}

We consider $V^{0}, p^{0}, V^{1}$ and $p^{1}$ defined in Theorem 1.1 , and we introduce $V^{2}:\left[0, T^{\star}\left[\times \mathcal{U} \longrightarrow \mathbb{R}^{3}\right.\right.$ such that :

$$
\left\{\begin{array}{l}
V^{2}=-\kappa\left(\frac{\partial V^{1}}{\partial n}\right)_{T} \text { on }\left[0, T^{\star}[\times \Gamma\right. \\
V^{2}=0 \text { on }\left[0, T^{\star}\right] \times \partial \Omega \\
\operatorname{div} V^{2}=0 \text { on }\left[0, T^{\star}\right] \times \mathcal{U}
\end{array}\right.
$$

and since $V^{1}$ satisfies regularity conditions given in Proposition 3.5 we can assume that :

$$
\left\{\begin{array}{l}
V^{2} \in L^{\infty}\left(0, T ; H^{4}(\mathcal{U})\right) \cap L^{2}\left(0, T ; H^{5}(\mathcal{U})\right) \\
\frac{\partial V^{2}}{\partial t} \in L^{\infty}\left(0, T ; H^{2}(\mathcal{U})\right) \cap L^{2}\left(0, T ; H^{3}(\mathcal{U})\right)
\end{array}\right.
$$

We recall that we consider $v^{\varepsilon}$ solution of the following problem :

$$
\begin{cases}\frac{\partial v^{\varepsilon}}{\partial t}-\Delta v^{\varepsilon}+\left(v^{\varepsilon} \cdot \nabla\right) v^{\varepsilon}+\nabla p^{\varepsilon}=f & \text { in }\left[0, T^{\star}[\times \mathcal{U}\right. \\ v^{\varepsilon}=0 & \text { in }\left[0, T^{\star}[\times \partial \Omega\right. \\ v^{\varepsilon} \cdot n=0 & \text { in }\left[0, T^{\star}[\times \Gamma\right. \\ v^{\varepsilon}=-\kappa \varepsilon\left(\frac{\partial v^{\varepsilon}}{\partial n}\right)_{T} & \text { in }\left[0, T^{\star}[\times \Gamma\right. \\ v^{\varepsilon}(0, x)=v_{0}^{\varepsilon}(x) & \text { in } \mathcal{U}\end{cases}
$$

We write the asymptotic expansion of $v^{\varepsilon}$ and $p^{\varepsilon}$ :

$$
\begin{aligned}
& v^{\varepsilon}=V^{0}+\varepsilon V^{1}+\varepsilon^{2} V^{2}+\varepsilon^{2} v_{\varepsilon}^{r} \\
& p^{\varepsilon}=p^{0}+\varepsilon p^{1}+\varepsilon^{2} p_{\varepsilon}^{r}
\end{aligned}
$$

The equation satisfied by the remainder term is the following :

$$
\begin{aligned}
& \frac{\partial v_{\varepsilon}^{r}}{\partial t}-\Delta v_{\varepsilon}^{r}+\varepsilon^{2}\left(v_{\varepsilon}^{r} \cdot \nabla\right) V^{\varepsilon}+\left(V^{\varepsilon} \cdot \nabla\right) v_{\varepsilon}^{r}+\nabla p_{\varepsilon}^{r}=g^{\varepsilon} \text { in } \mathcal{U} \\
& \operatorname{div} v_{\varepsilon}^{r}=0 \text { in } \mathcal{U} \\
& v_{\varepsilon}^{r}=-\kappa \varepsilon\left(\frac{\partial v_{\varepsilon}^{r}}{\partial n}\right)_{T}-\kappa \varepsilon\left(\frac{\partial V^{2}}{\partial n}\right)_{T} \text { on } \Gamma \\
& v_{\varepsilon}^{r} \cdot n=0 \text { on } \Gamma \\
& v_{\varepsilon}^{r}=0 \text { on } \partial \Omega
\end{aligned}
$$


where

$$
\begin{aligned}
g^{\varepsilon}= & -\frac{\partial V^{2}}{\partial t}+\Delta V^{2}-\left(V^{0} \cdot \nabla\right) V^{2}-\left(V^{1} \cdot \nabla\right) V^{1}-\left(V^{2} \cdot \nabla\right) V^{0} \\
& -\varepsilon\left(V^{1} \cdot \nabla\right) V^{2}-\varepsilon\left(V^{2} \cdot \nabla\right) V^{1}-\varepsilon^{2}\left(V^{2} \cdot \nabla\right) V^{2}
\end{aligned}
$$

We remark that

$$
g^{\varepsilon} \in L^{\infty}\left(0, T ; L^{2}(\mathcal{U})\right) \text { for all } T<T^{\star} .
$$

We multiply Equation (5.3) by $v_{\varepsilon}^{r}$. Since

$$
\begin{aligned}
& \int_{\mathcal{U}}\left(v_{\varepsilon}^{r} \cdot \nabla\right) v_{\varepsilon}^{r}=-\frac{1}{2} \int_{\Gamma}\left(v_{\varepsilon}^{r} \cdot n\right)\left|v_{\varepsilon}^{r}\right|^{2}=0 \\
& \int_{\mathcal{U}} \nabla p_{\varepsilon}^{r} \cdot v_{\varepsilon}^{r}=-\int_{\mathcal{U}} p_{\varepsilon}^{r} \operatorname{div} v_{\varepsilon}^{r}=0 \\
& -\int_{\Gamma} \frac{\partial v_{\varepsilon}^{r}}{\partial n} \cdot v_{\varepsilon}^{r}=\frac{1}{\varepsilon} \int_{\Gamma}\left|v_{\varepsilon}^{r}\right|^{2}+\int_{\Gamma}\left(\frac{\partial V^{2}}{\partial n}\right)_{T} \cdot v_{\varepsilon}^{r}
\end{aligned}
$$

and with the estimates on $g^{\varepsilon}$ and $V^{2}$, we obtain that for all $T<T^{\star}$ there exists $C$ such that :

$\frac{1}{2} \frac{d}{d t}\left\|v_{\varepsilon}^{r}\right\|_{L^{2}(\mathcal{U})}^{2}+\left\|\nabla v_{\varepsilon}^{r}\right\|_{L^{2}(\mathcal{U})}^{2}+\frac{1}{\varepsilon} \int_{\Gamma}\left|v_{\varepsilon}^{r}\right|^{2} \leq \frac{1}{2}\left\|\nabla v_{\varepsilon}^{r}\right\|_{L^{2}(\mathcal{U})}^{2}+C\left\|v_{\varepsilon}^{r}\right\|_{L^{2}(\mathcal{U})}^{2}+\frac{1}{2 \varepsilon} \int_{\Gamma}\left|v_{\varepsilon}^{r}\right|^{2}+\frac{\varepsilon}{2}\left\|\frac{\partial V^{2}}{\partial n}\right\|_{L^{2}(\Gamma)}$

that is for all $T<T^{\star}$ there exists a constant $K$ such that :

$$
\frac{d}{d t}\left\|v_{\varepsilon}^{r}\right\|_{L^{2}(\mathcal{U})}^{2}+\left\|\nabla v_{\varepsilon}^{r}\right\|_{L^{2}(\mathcal{U})}^{2}+\frac{1}{4 \varepsilon} \int_{\Gamma}\left|v_{\varepsilon}^{r}\right|^{2} \leq K+K\left\|v_{\varepsilon}^{r}\right\|_{L^{2}(\mathcal{U})}^{2}
$$

and we conclude the proof with a classical Gronwall Lemma.

\section{Appendix}

This appendix is devoted to the calculation of the differential operators in the geometry of thin layers.

\subsection{Local parametrization of $\Gamma$}

We consider a local parametrization of $\Gamma$ : let $U$ (resp. $V$ ) be an open subset of $\mathbb{R}^{2}$ (resp. $\mathbb{R}^{3}$ ) and let $X: U \longrightarrow V$ be a local regular parametrization of $V \cap \Gamma$.

We denote $\nu=n \circ X$

For $\left(u_{1}, u_{2}\right) \in U$, we denote $g(0)$ the matrix of the first fundamental form on $\Gamma$ with entries :

$$
g_{i j}(0)\left(u_{1}, u_{2}\right)=\left(\frac{\partial X}{\partial u_{i}} \cdot \frac{\partial X}{\partial u_{j}}\right)\left(u_{1}, u_{2}\right) .
$$

We denote $g^{i j}(0)$ the coefficents of the matrix $g(0)^{-1}$.

The matrix $b$ of the second fundamental form is the matrix with entries $b_{i j}$ given by :

$$
b_{i j}\left(u_{1}, u_{2}\right)=\left(\frac{\partial \nu}{\partial u_{i}} \cdot \frac{\partial X}{\partial u_{j}}\right)\left(u_{1}, u_{2}\right)
$$

We know that $g(0)$ and $b$ are symmetric. 
The map $n: \Gamma \longrightarrow S^{2}$ is regular and its derivate $d n(\sigma)$ is a linear map from $T_{\sigma} \Gamma$ into $T_{n(\sigma)} S^{2}$. Since $T_{\sigma} \Gamma=T_{n(\sigma)} S^{2}$, we can consider that $d n(\sigma)$ is an endomorphism of $T_{\sigma} \Gamma$.

We denote $d$ the matrix of $d n(\sigma)$ in the basis $\left(\frac{\partial X}{\partial u_{1}}, \frac{\partial X}{\partial u_{2}}\right)$, and we denote $d_{i j}$ its entries.

We have :

$$
\frac{\partial \nu}{\partial u_{j}}=d n(\sigma)\left(\frac{\partial X}{\partial u_{j}}\right)=\sum_{i} d_{i j} \frac{\partial X}{\partial u_{i}}
$$

hence, taking the scalar product with $\frac{\partial X}{\partial u_{k}}$ we have $b_{k j}=\sum_{i} d_{i j} g_{k i}(0)$ that is $b=g(0) d$.

\subsection{Calculus on $\Gamma$}

If $\tilde{v}: \Gamma \longrightarrow \mathbb{R}$ is regular, we set $\bar{v}=v \circ X$. We define the integral on $\Gamma$ and the differential operators on $\Gamma$ with the map $X$ :

$$
\int_{\Gamma \cap V} \tilde{v}(\sigma) d \sigma=\int_{U} \bar{v}\left(u_{1}, u_{2}\right) \sqrt{\operatorname{det} g(0)\left(u_{1}, u_{2}\right)} d u_{1} d u_{2}
$$

and

$$
\nabla_{\Gamma} \tilde{v}(\sigma)=\left(\sum_{i, j \in\{1,2\}} g^{i j}(0) \frac{\partial \bar{v}}{\partial u_{j}} \frac{\partial X}{\partial u_{i}}\right)\left(X^{-1}(\sigma)\right)
$$

In addition

$$
\Delta_{\Gamma} \tilde{v}(\sigma)=\frac{1}{\sqrt{\operatorname{det} g(0)}} \sum_{i, j} \frac{\partial}{\partial u_{i}}\left(g^{i j}(0) \frac{\partial \bar{v}}{\partial u_{j}} \sqrt{\operatorname{det} g(0)}\right)\left(X^{-1}(\sigma)\right) .
$$

Furthermore, if $\tilde{Z}: \Gamma \longrightarrow T \Gamma$ is a tangent vector field, we decompose $Z$ in the basis $\left(\frac{\partial X}{\partial u_{1}}, \frac{\partial X}{\partial u_{2}}\right)$ : $Z=\gamma_{1} \frac{\partial X}{\partial u_{1}}+\gamma_{2} \frac{\partial X}{\partial u_{2}}$ and we have :

$$
\operatorname{div}_{\Gamma} \tilde{Z}(\sigma)=\left(\frac{1}{\sqrt{\operatorname{det} g(0)}} \sum_{i=1}^{2} \frac{\partial}{\partial u_{i}}\left(\gamma_{i} \sqrt{\operatorname{det} g(0)}\right)\right)\left(X^{-1}(\sigma)\right) .
$$

Remark 6.1 One can verify that these definitions do not depend on the map $X$ (see $[11], \ldots)$

\subsection{Local parametrization of $\omega_{\eta_{0}}$.}

We define $Y$ by :

$$
\begin{aligned}
& Y: U \times] 0, \eta_{0}\left[\quad \longrightarrow \mathbb{R}^{3}\right. \\
& \left(u_{1}, u_{2}, u_{3}\right) \mapsto X\left(u_{1}, u_{2}\right)+u_{3} \nu\left(u_{1}, u_{2}\right)
\end{aligned}
$$

We denote $\hat{g}\left(u_{1}, u_{2}, u_{3}\right)$ the $(3,3)$ matrix of the scalar product in the new coordinates, with entries :

$$
\hat{g}_{i j}=\left(\frac{\partial Y}{\partial u_{i}} \cdot \frac{\partial Y}{\partial u_{j}}\right) .
$$

We remark that $\frac{\partial Y}{\partial u_{3}}\left(u_{1}, u_{2}, u_{3}\right)=\nu\left(u_{1}, u_{2}\right)$ and for $i \in\{1,2\}, \frac{\partial Y}{\partial u_{i}}=\frac{\partial X}{\partial u_{i}}\left(u_{1}, u_{2}\right)+u_{3} \frac{\partial \nu}{\partial u_{i}}\left(u_{1}, u_{2}\right)$. Since $\nu\left(u_{1}, u_{2}\right)$ is normal to $\Gamma$ at the point $X\left(u_{1}, u_{2}\right)$,

$$
\left(\nu\left(u_{1}, u_{2}\right) \cdot \frac{\partial X}{\partial u_{i}}\left(u_{1}, u_{2}\right)\right)=0
$$


Furthermore, since for all $\left(u_{1}, u_{2}\right),\left\|\nu\left(u_{1}, u_{2}\right)\right\|=1$,

$$
\left(\frac{\partial \nu}{\partial u_{i}}\left(u_{1}, u_{2}\right) \cdot \nu\left(u_{1}, u_{2}\right)\right)=0 .
$$

Thus the matrix $g$ is on the form :

$$
\hat{g}\left(u_{1}, u_{2}, s\right)=\left(\begin{array}{ccc}
g(s)\left(u_{1}, u_{2}\right) & 0 \\
0 & 0 & 1
\end{array}\right)
$$

where $g(s)\left(u_{1}, u_{2}\right)$ is the $(2,2)$ matrix with entries $g_{i j}(s)\left(u_{1}, u_{2}\right)$ defined by :

$$
g_{i j}(s)\left(u_{1}, u_{2}\right)=\left(\frac{\partial X}{\partial u_{i}}+s \frac{\partial \nu}{\partial u_{i}} \cdot \frac{\partial X}{\partial u_{j}}+s \frac{\partial \nu}{\partial u_{j}}\right)\left(u_{1}, u_{2}\right) .
$$

We denote $g^{i j}(s)$ the entries of the matrix $[g(s)]^{-1}$.

We remark that :

$$
\begin{aligned}
g_{i j}(s) & =g_{i j}(0)+2 s b_{i j}+s^{2}\left(\frac{\partial \nu}{\partial u_{i}} \cdot \sum_{k} d_{k j} \frac{\partial X}{\partial u_{k}}\right) \\
& =g^{i j}(0)+2 s b_{i j}+s^{2} \sum_{k} b_{i k} d_{k j}
\end{aligned}
$$

Thus

$$
g(s)=g(0)+2 s b+s^{2} b d .
$$

Remark 6.2 For $s \in] 0, \eta_{0}\left[\right.$, the map $X_{s}$ defined by $X_{s}\left(u_{1}, u_{2}\right)=Y\left(u_{1}, u_{2}, s\right)$ is a local parametrization of $\Gamma_{s}$, and $g(s)\left(u_{1}, u_{2}\right)$ is the matrix of the first fundamental form of $\Gamma_{s}$.

\subsection{Integration in the new coordinates}

For $\sigma \in \Gamma \cap V$, if $\sigma=X\left(u_{1}, u_{2}\right)$, we define $\gamma_{s}(\sigma)$ by :

$$
\gamma_{s}(\sigma)=\left[\left(\operatorname{det} g^{-1}(0) g(s)\right)\left(u_{1}, u_{2}\right)\right]^{\frac{1}{2}} .
$$

Proposition 6.1 $\gamma_{s}$ does not depend on the parametrization of $\Gamma$ since we have :

$$
\gamma_{s}(\sigma)=\operatorname{det}(I d+s d n)(\sigma)
$$

Proof : we have :

$$
g^{-1}(0) g(s)=I d+2 s g^{-1}(0) b+s^{2} g^{-1}(0) b d
$$

and since $d=g^{-1}(0) b$, we obtain that

$$
g^{-1}(0) g(s)=I d+2 s d+s^{2} d^{2}=(I d+s d)^{2} .
$$

Proposition 6.2 If $v: \omega_{\eta_{0}} \longrightarrow \mathbb{R}$, denoting $\tilde{v}=v \circ \Psi$, we have :

$$
\int_{\omega_{\eta_{0}}} v=\int_{0}^{\kappa \eta_{0}} \int_{\Gamma} \tilde{v}(\sigma, s) \gamma_{s}(\sigma) d \sigma d s
$$


Proof : using an atlas covering $\Gamma$ and a partition of the unity we may consider $u$ with support in $Y(U \times] 0, \kappa \eta_{0}[)$. Then we have :

$$
\begin{aligned}
\int_{\omega_{\eta_{0}}} v & =\int_{U \times] 0, \kappa \eta_{0}[} v \circ Y \sqrt{\operatorname{det} \hat{g}} \\
& =\int_{0}^{\kappa \eta_{0}} \int_{U} \tilde{v}\left(X\left(u_{1}, u_{2}\right), s\right) \sqrt{\operatorname{det} g_{s}\left(u_{1}, u_{2}\right)} d u_{1} d u_{2} d s \\
& =\int_{0}^{\kappa \eta_{0}} \int_{U} \tilde{v}\left(X\left(u_{1}, u_{2}\right), s\right) \gamma_{s}\left(X\left(u_{1}, u_{2}\right)\right) \sqrt{\operatorname{det} g_{0}\left(u_{1}, u_{2}\right)} d u_{1} d u_{2} d s \\
& =\int_{0}^{\kappa \eta_{0}} \int_{\Gamma} \tilde{v}(\sigma, s) \gamma_{s}(\sigma)|d \sigma| d s .
\end{aligned}
$$

\subsection{Gradient in the new coordinates}

For $s \in\left[0, \kappa \eta_{0}\right]$ and for $\tilde{w}: \Gamma \longrightarrow \mathbb{R}$ we define $\nabla_{\Gamma_{s}} \tilde{w}$ by :

$$
\nabla_{\Gamma_{s}} \tilde{w}(\sigma)=(I d+\operatorname{sdn}(\sigma))^{-1} \nabla_{\Gamma_{0}} \tilde{w}(\sigma) .
$$

We have the following lemma :

Lemma 6.1 Let $v: \omega_{\eta_{0}} \longrightarrow \mathbb{R}$. We denote $\tilde{v}=v \circ \Psi$. For $x \in \omega_{\eta_{0}}$ we have

$$
\nabla v(x)=\frac{\partial \tilde{v}}{\partial z}(P(x), \varphi(x)) n(P(x))+\left(\nabla_{\Gamma_{\varphi(x)}} \tilde{v}\right)(P(x), \varphi(x)) .
$$

Proof : we set $\bar{v}=v \circ Y$.

In the coordinates $\left(u_{1}, u_{2}, u_{3}\right)$ we have :

$$
\nabla v(x)=\left(\sum_{i=1}^{3}\left(\sum_{j} \hat{g}^{i j} \frac{\partial \bar{v}}{\partial u_{j}}\right) \frac{\partial Y}{\partial u_{i}}\right)\left(Y^{-1}(x)\right)
$$

where $\hat{g}^{i j}$ are the entries of $\hat{g}^{-1}$. We remark that for $i=3$,

$$
\left(\sum_{j} \hat{g}^{3 j} \frac{\partial \bar{v}}{\partial u_{j}}\right)=\frac{\partial \bar{v}}{\partial u_{3}}
$$

Furthermore, $\frac{\partial Y}{\partial u_{3}}\left(u_{1}, u_{2}, u_{3}\right)=\nu\left(u_{1}, u_{2}\right)$.

In addition, for $i \in\{1,2\}, \hat{g}^{i 3}=\hat{g}^{3 i}=0$ hence

$$
\sum_{j=1}^{3} \hat{g}^{i j} \frac{\partial \bar{v}}{\partial u_{j}}=\sum_{j=1}^{2} g^{i j}(s) \frac{\partial \bar{v}}{\partial u_{j}} .
$$

Now we remark that

$$
\nabla_{\Gamma_{s}} \tilde{v}=\sum_{i=1}^{2}\left(\sum_{j=1}^{2} g^{i j}(s) \frac{\partial \bar{v}}{\partial u_{j}}\right) \frac{\partial Y}{\partial u_{i}}
$$


Proof : we know that $g^{-1}(0) g(s)=(I d+s d)^{2}$ thus denoting $\alpha=I d+s d$, and $\alpha_{i j}$ (resp. $\left.\alpha^{i j}\right)$ the entries of the matrix $\alpha$ (resp. $\alpha^{-1}$ ), we have :

$$
g^{-1}(s)=\alpha^{-2} g^{-1}(0)
$$

so

$$
g^{i j}(s)=g^{j i}(s)=\sum_{k, l} \alpha^{i k} \alpha^{k l} g^{l j}(0)
$$

Thus

$$
\begin{aligned}
\sum_{i=1}^{2}\left(\sum_{j=1}^{2} g^{i j}(s) \frac{\partial \bar{v}}{\partial u_{j}}\right) \frac{\partial Y}{\partial u_{i}} & =\sum_{i j} \frac{\partial \bar{v}}{\partial u_{j}} g^{i j}(s)\left(\sum_{p} \alpha_{p i} \frac{\partial X}{\partial u_{p}}\right) \\
& =\sum_{i, j, k, l, p} \frac{\partial \bar{v}}{\partial u_{j}} \alpha^{i k} \alpha^{k l} g^{l j}(0) \alpha_{p i} \frac{\partial X}{\partial u_{p}}
\end{aligned}
$$

and since $\sum_{i} \alpha^{i k} \alpha_{p i}=\delta_{k p}$ we have :

$$
\begin{aligned}
\sum_{i=1}^{2}\left(\sum_{j=1}^{2} g^{i j}(s) \frac{\partial \bar{v}}{\partial u_{j}}\right) \frac{\partial Y}{\partial u_{i}} & =\sum_{j, l, p} \frac{\partial \bar{v}}{\partial u_{j}} \alpha^{p l} g^{l j}(0) \alpha_{k p} \frac{\partial X}{\partial u_{p}} \\
& =\sum_{j l} \frac{\partial \bar{v}}{\partial u_{j}} g^{l j}(0)\left(\sum_{p} \alpha^{p l} \frac{\partial X}{\partial u_{p}}\right) \\
& =(I d+s d n(\sigma))^{-1}\left(\sum_{j l} \frac{\partial \bar{v}}{\partial u_{j}} g^{l j}(0) \frac{\partial X}{\partial u_{l}}\right) \\
& =(I d+s d n(\sigma))^{-1} \nabla_{\Gamma} \tilde{v} \\
& =\nabla_{\Gamma_{s}} \tilde{v} .
\end{aligned}
$$

\subsection{Divergence in the new coordinates}

For $\tilde{W}: \Gamma \longrightarrow T \Gamma$ a tangent vector field, and for $s \in\left[0, \kappa \eta_{0}\right]$ we define $\operatorname{div}_{\Gamma_{s}}$ by :

$$
\operatorname{div}_{\Gamma_{s}} \tilde{W}=\frac{1}{\gamma_{s}} \operatorname{div}_{\Gamma}\left(\gamma_{s}(I d+s d n)^{-1} \tilde{W}\right) .
$$

Furthermore we denote $G_{s}(\sigma)=\left(\frac{1}{\gamma_{s}} \frac{\partial \gamma_{s}}{\partial s}\right)(\sigma)$.

Lemma 6.2 If $Z: \omega_{\eta_{0}} \longrightarrow \mathbb{R}^{3}$, we denote $\widetilde{Z}=Z \circ \Psi$. We define the normal and the tangential parts of $Z$ by :

$$
\begin{aligned}
& \widetilde{Z}_{N}(\sigma, z)=\widetilde{Z}(\sigma, z) \cdot n(\sigma), \\
& \widetilde{Z}_{T}(\sigma, z)=\widetilde{Z}(\sigma, z)-\widetilde{Z}_{N}(\sigma, z) n(\sigma) .
\end{aligned}
$$

Then we have :

$$
\operatorname{div} Z(x)=\frac{\partial \widetilde{Z}_{N}}{\partial z}(P(x), \varphi(x))+G_{\varphi(x)}(P(x)) \widetilde{Z}_{N}(P(x), \varphi(x))+\left(\operatorname{div}_{\Gamma_{\varphi(x)}} \widetilde{Z}_{T}\right)(P(x), \varphi(x))
$$


Proof : by duality we will obtain the expression of the divergence operator in the new coordinates :

$$
\begin{aligned}
\int_{\omega_{\eta_{0}}} v \operatorname{div} Z & =-\int_{\omega_{\eta_{0}}} Z \cdot \nabla v \\
& =-\int_{0}^{\kappa \eta_{0}} \int_{\Gamma} \tilde{Z} \cdot \nabla \tilde{v} \gamma_{s} \\
& =-\int_{0}^{\kappa \eta_{0}} \int_{\Gamma}\left(\tilde{Z}_{N} \frac{\partial \tilde{v}}{\partial z}+\left(\tilde{Z}_{T} \cdot \nabla_{\Gamma_{s}} \tilde{v}\right)\right) \gamma_{s} \\
& =-\int_{0}^{\kappa \eta_{0}} \int_{\Gamma} \tilde{Z}_{N} \frac{\partial \tilde{v}}{\partial z} \gamma_{s}-\int_{0}^{\kappa \eta_{0}} \int_{\Gamma}\left(\gamma_{s}(I d+s d n)^{-1} \tilde{Z}_{T} \cdot \nabla_{\Gamma_{0}} \tilde{v}\right)
\end{aligned}
$$

since $I d+s d n$ is a symetric operator of $T_{\sigma} \Gamma$.

Integrating by part we obtain that :

$$
\int_{\omega_{\eta_{0}}} v \operatorname{div} Z=\int_{0}^{\kappa \eta_{0}} \int_{\Gamma}\left(\frac{1}{\gamma_{s}} \frac{\partial}{\partial z}\left(\tilde{Z}_{N} \gamma_{s}\right)+\frac{1}{\gamma_{s}} \operatorname{div}{ }_{\Gamma}\left(\gamma_{s}(I d+s d n)^{-1} \tilde{Z}_{T}\right)\right) \tilde{v} \gamma_{s}
$$

wich concludes the proof of the lemma.

\subsection{Laplace operator in the new coordinates}

Let $u: \omega_{\eta_{0}} \longrightarrow \mathbb{R}$. We denote $\tilde{u}=u \circ \Psi$.

Since $\Delta=\operatorname{div} \nabla$ we obtain that :

$$
\Delta u(x)=\frac{\partial^{2} \tilde{u}}{\partial z^{2}}(P(x), \varphi(x))+G_{\varphi(x)}(P(x)) \frac{\partial \tilde{u}}{\partial z}(P(x), \varphi(x))+\left(\Delta_{\Gamma_{\varphi(x)}} \tilde{u}\right)(P(x), \varphi(x))
$$

with $\Delta_{\Gamma_{s}} \tilde{u}=\operatorname{div} \Gamma_{\Gamma_{s}}\left(\nabla_{\Gamma_{s}} \tilde{u}\right)$.

Example : if $\Gamma$ is the unit sphere of $\mathbb{R}^{3}$, we take the classical parametrization of $S^{2}$ :

$$
X:(\theta, \varphi) \mapsto(\cos \theta \cos \varphi, \cos \theta \sin \varphi, \sin \theta) .
$$

We denote $e_{\varphi}=(-\sin \varphi, \cos \varphi, 0)$ and $e_{\theta}=(-\sin \theta \cos \varphi,-\sin \theta \sin \varphi, \cos \theta)$.

Let $\tilde{v}: S^{2} \longrightarrow \mathbb{R}$. We introduce $\bar{v}=\tilde{v} \circ X$. Then :

$$
\begin{gathered}
\nabla_{\Gamma_{s}} \tilde{v}=\left(\frac{1}{1-s} \frac{\partial \bar{v}}{\partial \theta}\right) e_{\theta}+\left(\frac{1}{(1-s) \cos \theta} \frac{\partial \bar{v}}{\partial \varphi}\right) e_{\varphi}, \\
\Delta_{\Gamma_{s}} \tilde{v}=\frac{1}{(1-s)^{2} \cos ^{2} \theta}\left(\cos \theta \frac{\partial}{\partial \theta}\left(\cos \theta \frac{\partial \bar{v}}{\partial \theta}\right)+\frac{\partial^{2} \bar{v}}{\partial \varphi^{2}}\right) .
\end{gathered}
$$

If $Z: \Gamma \longrightarrow T \Gamma$, we decompose $Z=Z_{\theta} e_{\theta}+Z_{\varphi} e_{\varphi}$ and we have :

$$
\operatorname{div}_{\Gamma_{s}} Z=\frac{1}{(1-s) \cos \theta}\left(\frac{\partial}{\partial \theta}\left(Z_{\theta} \cos \theta\right)+\frac{\partial Z_{\varphi}}{\partial \varphi}\right) .
$$

\section{Acknowledgements}

The author wishes to thank P. Fabrie and C. H. Bruneau for many stimulating discussions. 


\section{References}

[1] Ph. Angot, Analysis of singular perturbation on the Brinkman problem for fictitious domain models of viscous flow, Math. Methods Appl. Sci. 22 (1999), 1395-1412.

[2] Ch.-H. Bruneau, Numerical Simulation and Analysis of the Transition to Turbulence, Lecture Notes in Physics, 490 (1997).

[3] Ch.-H. Bruneau, P. Fabrie, Effective downstream boundary conditions for incompressible navier-Stokes equations, Int. J. Numer. Methods in Fluids, 19 (1994), 693-705.

[4] Ch.-H. Bruneau, P. Fabrie, New efficient boundary conditions for incompressible navierStokes equations: a well-posedness result, M2AN , 30 (1996), 815-840.

[5] Ch.-H. Bruneau, I. Mortazavi, Contrôle passif d'écoulements incompressibles autour d'obstacles à l'aide de milieux poreux, CRAS, 329 série IIb, 2001.

[6] V. Bruneau, G. Carbou, Spectral asymptotic in the large coupling limit, Asymptotic Analysis 29 (2002), 91-113.

[7] G. Carbou, P. Fabrie, Boundary layer for a penalization method for viscous incompressible flow, submited.

[8] G. Carbou, P. Fabrie, O. Guès, Couche limite en micromagnetisme, Comm. Partial Differential Equations 27 (2002), 1467-1495.

[9] L. Cattabriga, Su un problema al contorno relativo al sistema di equazioni di Stokes, Rend. Semin. Mat. Univ. Padova, 31 (1961), 308-340.

[10] V. Giraud, P. A. Raviart, Finite element methods for Navier-Stokes equations. Theory and algorithms. Springer Series in Computational Mathematics, 5. Springer-Verlag, Berlin, 1986.

[11] F. Hélein, Harmonic maps, conservation laws and moving frames. Translated from the 1996 French original. With a foreword by James Eells. Second edition. Cambridge Tracts in Mathematics, 150. Cambridge University Press, Cambridge, 2002.

[12] K. Khadra, S. Parneix, Ph. Angot, J.P. Caltagirone, Fictitious domain approach for numerical modeling of Navier-Stokes equation, Int. J. Numer. Meth. in Fluids, 34 (2000), $651-684$.

[13] O.A. Ladyzhenskaya, The boundary value problems of mathematical physics. Translated from the Russian by Jack Lohwater [Arthur J. Lohwater]. Applied Mathematical Sciences, 49. Springer-Verlag, New York, 1985.

[14] A. Mikelic, Stationary incompressible viscous fluid flow through a porous boundary. Z. Angew. Math. Mech. 67 (1987), 273-275

[15] R. Temam, M. Ziane, Navier-Stokes equations in three-dimensional thin domains with various boundary conditions, Adv. Differential Equations 1 (1996), 499-546. 